\title{
From Alpha Diversity to ZZZ: Exploring associations among sleep, gut bacteria and behavioral development in infancy
}

Schoch, S.F. ${ }^{1,2}$., Castro-Meija, J.L. ${ }^{3}$, Krych, L., Kot ${ }^{3}$, W., Leng, B. ${ }^{3}$, Kohler, M. ${ }^{1}$, Huber, R. ${ }^{4,5}$, Rogler, G. ${ }^{6}$, Biedermann, L. ${ }^{6}$, Walser, JC. ${ }^{7}$, Nielsen, D.S. ${ }^{3}$, Kurth, S. ${ }^{1,8}$

1) Department of Pulmonology, University Hospital Zurich, Zurich, $\mathrm{CH}$

2) University of Zurich, Zurich, $\mathrm{CH}$

3) Department of Food Science, University of Copenhagen, DK

4) Child Development Center, University Children's Hospital Zurich, Zurich, $\mathrm{CH}$

5) Department of Child and Adolescent Psychiatry and Psychotherapy, Psychiatric Hospital, University of Zurich, $\mathrm{CH}$

6) Department for Gastroenterology and Hepatology, University Hospital Zurich, Zurich, $\mathrm{CH}$

7) Genetic Diversity Center, ETH Zurich, Zurich, $\mathrm{CH}$

8) Department of Psychology, University of Fribourg, Fribourg, $\mathrm{CH}$

\section{Abstract}

Infancy is a period of marked development, entailing many maturation processes that lay the foundation for later health. Two crucial health factors in infancy are the establishment of a sleep-wake rhythm and the growth of gut bacterial diversity - both have been linked to later cognitive and physiological outcomes. In adults and animal models, evidence is emerging that sleep and gut bacteria are bidirectionally linked. However, it is yet unclear when and how the sleep-gut link in early life evolves. Here we demonstrate that a sleep-gut link develops in infancy, undergoes transitional dynamics, and involves an early sensitive period. Specifically, infants' daytime sleep is linked to markers of bacterial diversity, and nighttime awakenings are associated with gut bacterial maturity and bacterial enterotype. Furthermore, both sleep and gut bacteria are associated with behavioral developmental outcomes, with the strongest connections at 3 months of age. Our results demonstrate the dynamic interplay of sleep and gut bacteria within healthy human development. As both sleep and gut bacteria are modifiable, they are promising targets for early intervention in clinical groups. 


\section{Introduction}

One in six children is affected by a developmental disorder (e.g., autism, developmental delays) [1]. Early recognition of such disorders is crucial to provide effective support and prevent aggravated consequences [2]. Two potential health targets in early childhood that can serve as protective factors are sleep behavior and gut bacteria.

Sleep regulation undergoes significant maturation in early development, with the most drastic changes in the first year of life. Although sleep behavior is highly variable between infants, universal maturational patterns are visible: in the first year of infancy, a 24-h rhythm emerges, and nighttime sleep is consolidated [3,4]. In the past decade, empirical work has revolutionized our understanding: from sleep as a global behavior towards sleep as a localized neurophysiological and cellular recovery process that interacts with neurodevelopment $[5,6]$. Sleep neurophysiology is assessed with the electroencephalogram (EEG) and unfolds throughout early childhood. This evolution entails not only a pronounced decrease of the fraction of rapid eye movement (REM) sleep [7] but, furthermore, the emergence of core sleep features such as sleep spindles [8-10] and slow waves [11,12]. Sleep and brain development are closely linked: slow waves mirror the underlying brain maturation processes [13-15]. Excitingly, the sleep-brain link goes beyond pure correlative observations: short, fragmented, or poorly consolidated sleep in infancy predicts later cognitive and psychosocial problems [16-19] (but see [20-22]), supporting now in young humans the demonstrated concept that the formation of neuronal connections necessitates adequate sleep [5,23-25].

Beyond sleep, we have come to understand the influence gut bacteria exert on the brain (for a comprehensive review, see [26]). Seminal research with germ-free mice, which lack this microbial ecosystem in the digestive tract, illuminates the bottom-up processes through which gut bacteria affect the brain [27-29]. Newly emerging insights on the gut-brain axis illuminate gut bacterial influences [30], how they promote stress regulation and mental health [31-33].

Early bacterial colonization is crucial because it is likely connected with the initiation of signaling processes in the brain. For example, germ-free mice have an 
exaggerated stress response compared to the control group of specific pathogenfree mice. Interestingly, the restoration of gut bacteria can partly reverse the stress response - yet exclusively in young but not in adult germ-free mice [34,35]. In alignment, compelling evidence is now emerging that gut bacteria also play a pivotal role in human brain development: The composition and diversity of bacterial taxa in infants' gut - quantified from stool samples - are linked to physiological growth and cognitive outcomes later in life [36]. Preliminary correlates between individual infant gut bacterial profiles with regional gray matter maturation support the gut-brain concept in infancy [37]. However, it remains unclear if and how sleep and gut bacteria jointly act throughout the process of neurodevelopment.

In adult humans, specific gut bacteria profiles have been associated with sleep disturbances, sleep quality, and sleep duration [38-40]. Both the timing and the duration of sleep influence gut bacteria [41-49] (yet [50]). In rodents, experimentally depleting gut bacteria specifically affects slow-wave sleep [51] - the most established proxy of sleep depth, and at the same time, the most conceptualized driver of neurodevelopment $[13,52]$. Crucially, a large fraction of gut bacterial taxa undergoes circadian rhythmicity influenced by the host circadian rhythm [53-55].

Interestingly, probiotics intake alleviated subjective and objective sleep disturbances related to stress in humans [56]. The sleep-gut-link may, therefore, be bi-directional. In strong support of this idea, experimental alterations of gut bacteria were shown to modify sleep consolidation in mice and rats [57-59].

It is unclear when the sleep-gut link starts to develop and when its maturation is completed. Yet, it is likely that a sleep-gut-brain link exists already in infancy, considering the sleep-neurodevelopment link, the abovementioned gut-brain axis together with the new evidence for a sleep-gut-brain link from investigations in adult humans and rodents. Importantly, the sequence of sensitive periods for brain development may pinpoint the sleep-gut link to be most pronounced, when the brain is most susceptible to external influences and thus most plastic in its connectivity. The early establishment of the sleep-gut link may therefore indeed determine later behavioral-developmental outcomes. 
This study examines whether a sleep-gut link exists in infancy and how its dynamics evolve across the first year of life. We hypothesized that more mature sleep-wake behavior patterns are linked to more mature infant gut bacteria profiles, the latter represented by the three measures: gut bacterial diversity, bacterial maturation index, and the assignment to enterotypes. To capture sleep maturation in great detail, we included the five primary infant sleep composites (Sleep Activity, Sleep Timing, Sleep Night, Sleep Day, and Sleep Variability) aligned with the sleep composites reported by Staples et al. (2019) [60]. Additionally, we expected a positive predictive effect of early maturity of sleep patterns and gut bacteria on later behavioral developmental status.

\section{METHODS}

\section{Participants}

162 healthy Swiss infants (75 female) participated in a study in which the three domains sleep, gut bacteria, and behavioral development were longitudinally investigated. We recruited infant participants with good general health, who were primarily breastfed, vaginally and term-born (37 - 43 week gestation week), and who experienced no antibiotic intake in their first three months of life (for detailed inclusion and exclusion criteria see [4]). Sub-sections of this dataset were published previously $[4,61]$. Ethical approval was obtained from the cantonal ethics committee (BASEC 2016-00730), and study procedures were consistent with the declaration of Helsinki. Written parental consent was obtained after explanation of the study and before enrollment. Families received small non-monetary gifts for their participation.

\section{Experimental design}

At infant age 3, 6, and 12 months, we quantified the three domains age-appropriately with validated objective and subjective methods (see Figure 1). At age 24 months, we conducted a follow-up assessment of behavioral development.

We measured infant sleep with ankle actigraphy and a 24-h-diary [61,62] for 11 continuous days. Parents attached the GENEactiv movement sensors (Activinsights Ltd, Kimbolton, UK, 43×40x13 mm, MEMS sensor, $16 \mathrm{~g}, 30 \mathrm{~Hz}$ Frequency) on the 
infants' left ankle using a modified sock or a Tyvek paper strap. We instructed parents to only remove the actigraph for bathing/swimming activities and document actigraph removal in the 24-h diary.

During each of the three assessments, parents collected at least one stool sample from the infant's diaper using disposable pipettes (Pastette $3 \mathrm{ml}$ graduated) or disposable laboratory spatulas (smartSpatula, $210 \mathrm{~mm}$ natural). Samples were kept in sterile Eppendorf tubes $(5 \mathrm{ml})$, wrapped into Whirlpak bags, and temporarily stored in the families' fridge. Samples were then transported to the laboratory within $48 \mathrm{~h}$ in cooling boxes to maintain temperature $(n=19$ samples were transported within 72 h). Aliquots with a minimum weight of $100 \mathrm{mg}$ were sampled and stored at $-50^{\circ}$ Celsius.

Infant behavioral developmental status was quantified with a parent-completed German translation of the Ages and Stages Questionnaire [63]. Using online questionnaires, we assessed feeding practices, sleep habits, general health, and demographics.

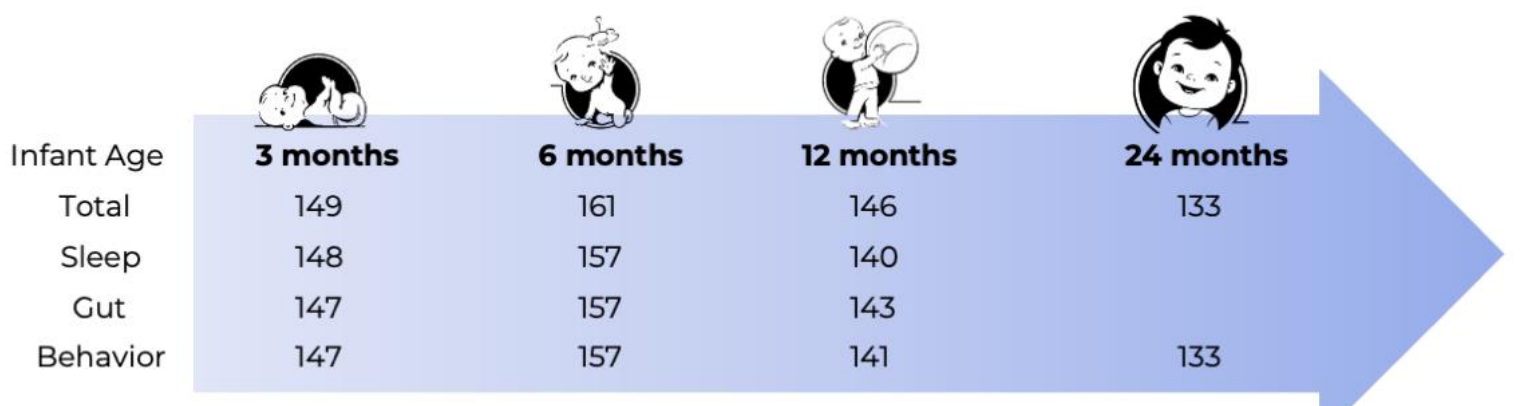

Figure 1. Experimental design to investigate the longitudinal interrelationships of sleep, gut bacteria, and behavioral development in healthy infants. Sleep entailed the collection of actigraphy and 24-h diary data [61], gut bacteria were characterized with 16S rRNA analysis from fecal samples, and behavior was quantified with the parent-reported Ages and Stages Questionnaire [63]. The sample size is indicated for each assessment in the corresponding domain. Graphics from Natalia Zelenina $\odot$ 123RF.com. 


\section{Missing Data}

10 infants were exclusively recruited for the 6 months assessment. Additionally, some infants were excluded for single assessment time points ( $\mathrm{n}=2$ at 3 months due to medication intake, $n=1$ at 6 months due to health reasons and $n=6$ at 12 months due to participant attrition and illness). Furthermore, 11 infants lacked sleep data for single assessment time points ( $n=1$ at 3 months due to holidays, $n=4$ at 6 months due to device failure (3) and withdrawal from sleep data collection (1), $n=6$ at 12 months due to device failure (2), moving away (1), prolonged illness (1), and withdrawal from sleep data collection (2)). Gut bacteria data was lacking from 9 infants for single assessment time points ( $n=2$ at 3 months due to low read count (1) and holidays (1), $n=4$ at 6 months due to low read count, $n=3$ at 12 months due to moving away (1), low read count (1) and sample not analyzed (1)). Behavioral data was missing from 11 infants for single assessment time points ( $n=2$ at 3 months and $n=4$ at 6 months and $n=5$ at 12 months of age all due to completion of questionnaire at an age outside of the selected time window). Additionally, only single recording days of sleep data were missing [4] or single items were not marked in survey. Missing data was replaced with 100 imputations using the R package mice [4] for sleep as well as gut bacteria outcome (i.e., alpha diversity, enterotype, random forest classification - methodological details follow), yet not for the intermediate quantification of gut bacterial counts.

\section{SLEEP}

\section{Actigraphy analysis}

Actigraph data were processed according to our laboratory standards [61]. We computed infant sleep-wake patterns with a 6-step modification of the Sadeh algorithm $[4,61,64]$. We calculated 48 sleep variables of interest, which were based on the recommendation of Meltzer et al. (2012) and Staples et al. (2019), and added a sleep regularity index $[60,65,66]$. To comprehensively capture all aspects of infant sleep maturation and at the same time reducing the number of analyses, we computed "sleep composites" [4]. A principal component analysis implemented a data-driven, similarity-based way: the 32 of the 48 sleep variables were consolidated into five sleep composites, which ultimately contained the key dimensions of infant sleep:

- Sleep Activity, summarizing movement and awakenings at night, 
- Sleep Timing, representing the clock time of bedtimes and sleep times,

- Sleep Night, reflecting nighttime sleep opportunity and duration,

- Sleep Day, characterizing duration and number of daytime naps and their regularity, and

- Sleep Variability, reflecting the variability of timing and nighttime sleep between the recorded days.

\section{GUT BACTERIA}

\section{Stool DNA extraction and 16s rRNA-gene amplicon sequencing}

DNA was extracted from $\sim 200 \mathrm{mg}$ of stool using PowerSoil[ DNA Isolation Kit (MOBIO Laboratories, Carlsbad, CA, USA) and following the manufacturer's instructions, but with minor modifications. Before DNA extraction, samples were placed into the PowerBead tubes and heat-treated at $65^{\circ} \mathrm{C}$ for $10 \mathrm{~min}$ and then at $95^{\circ} \mathrm{C}$ for $10 \mathrm{~min}$. Subsequently, solution $\mathrm{C} 1$ was added, and bead-beating was performed in FastPrep (MP Biomedicals, Santa Ana, CA, USA) using three cycles of $15 \mathrm{~s}$ each, at a speed of $6.5 \mathrm{~m} \mathrm{~s}^{-1}$. The remaining DNA extraction procedure followed the manufacturer's instructions.

NextSeq-based 16S rRNA gene amplicons were sequenced from the $\mathrm{V} 3$ region [67] using primers designed with adapters for the Nextera Index Kit ${ }^{\text {(Illumina, }} \mathrm{CA}$, USA): NXt_338_F: 5'- TCG TCG GCA GCG TCA GAT GTG TAT AAG AGA CAG ACW CCT ACG GGW GGC AGC AG -3' and NXt_518_R: 5'- GTC TCG TGG GCT CGG AGA TGT GTA TAA GAG ACA GAT TAC CGC GGC TGC TGG -3'.

Amplification profile ( $\left.1^{\text {st }} P C R\right)$, barcoding ( $\left.2^{\text {nd }} P C R\right)$, amplicon library purification, and sequencing were performed as previously described [68]. The raw dataset containing $2 \times 151 \mathrm{bp}$ (pair-ended) was quality checked using FastQC (v.0.11.2) [69]. Overlapping forward and reverse reads were trimmed (R1:5bp, R2:10bp) using seqtk [70] and merged using FLASH (v1 .2.11) [71] with the following parameters: minimum overlap of 15 , a maximum overlap of 300 , and maximum mismatch density of 0.25 . Cutadapt (v1.12) [72] was used to identify and trim primer regions allowing for an error rate of 0.01. Quality filtering was done using PrinSeq [73], removing reads with mean quality lower than 20 or containing ambiguous nucleotides. The resulting amplicons were de-noised and assigned to define Operational Taxonomic Units 
(OTUs) at 97\% sequence identity using usearch (v10.0.240, UNOISE3) [74].

Taxonomic predictions were made using SINTAX [75] and the Greengenes catalog [76].

\section{Analysis of gut bacteria data}

Samples were analyzed in five batches, across which the variability in sequencing depth was computed. To account for possible differences in sequencing depth and gut bacteria composition and diversity between batches, 'Batch' was included in all further analyses as a control variable. Samples below 50'000 reads (< $1 \%$ of all samples) were excluded to ensure ample sequencing depth (leading to $n=6$ missing, as reported above). Data were normalized by rarefying to the lowest count number (50'268), resulting in 1'430 bacterial taxa.

Ordination plots based on bray distance were inspected for quality-check by uncovering possible sample irregularities due to mother and infant antibiotic use or sickness. Although the vast majority of these flagged samples were within the normal range, we nonetheless computed a reduced data database with exclusion of six potential outliers (sickness $n=2$, maternal antibiotic use $n=3$, infant antibiotic use $n=1)$. Results with this reduced dataset are specifically referred to in the text. Negative controls (water probes) also contained reads (range 2 - 150'785). ZOTU2 (Enterobacteriaceae) were the abundant OTUs in $30 \%$ of negative controls and in $10 \%$ of the effective infant stool samples. Other OTUs, which were abundantly detected in the negative controls, were rare in the effective samples. The influence of ZOTU2 on the three bacterial markers (gut bacterial diversity, bacterial maturation index, enterotype) was negligible.

We defined the core bacteria by the fulfilling of two criteria across all time points: bacterial taxa reaching a minimal prevalence of $20 \%$ (i.e., detected in at least $20 \%$ of infants) and a minimal relative abundance of $1 \%$ (i.e., accounting for at least $1 \%$ of all bacterial measured in total). Following this preprocessing, we used three markers to streamline the quantification of gut bacterial profiles:

i) Gut bacterial diversity: characterizing the presence and abundance of bacterial taxa within individual samples (alpha diversity),

ii) Bacterial maturation index: reflecting the relative maturational status of each individuals' gut bacterial profile computed through the difference 
between random forest prediction of chronological age and actual chronological age,

iii) “Enterotypes”: classifying individuals' gut bacterial profiles and assignment to groups with similar typical bacterial representatives.

For i), we computed three measures to thoroughly capture alpha diversity:

Observed, Shannon, and Chao1. Observed emphasizes the number of different bacterial species present in a sample; Shannon concentrates on both - species abundance and evenness (similarity of abundance between species), and Chao1 is an abundance estimator with a focus on rare species [77].

For ii), random forest analysis was performed to predict chronological age from the bacterial composition in each stool profile (predicted bacterial age) [78]. We aimed to identify those infants demonstrating a mismatch between actual and predicted bacterial age. Therefore, we selected the comparatively low number of 100 trees in random forest analysis and then calculated a bacterial maturation index: "predicted bacterial age - actual age".

For iii), we first applied clustering scoring methods (prediction strength, average silhouette width, and Calinksi-Harabasz scoring) to assess the ideal number of clusters (2 to 12) and distance measure (Bray-Curtis, Jaccard, Unifrac, weighted Unifrac, and Jensen-Shannon Distance) and 2 to 12 clusters [79]. Most support was found for a 2-cluster solution with weighted Unifrac. Based on this solution, each infant at each assessment time point was assigned to either enterotype $A$ or B. Additionally, we examined the change in enterotype evolution pattern across the first year by classifying infants to "Switchers", "Bifidobacterium", "Bacteroides" and "Reverser".

\section{BEHAVIORAL DEVELOPMENTAL STATUS}

\section{Ages and Stages Questionnaire}

To quantify behavioral developmental status, parents completed a German translation of the Ages and Stages questionnaire, appropriate for the respective infant age [63]. We calculated a Collective Score for overall development by assembling the five key domains Communication, Gross Motor, Fine Motor, Problem Solving, and Personal Social. In addition to the overall Collective Score, we examined Gross Motor and Personal Social independently [63,80,81]. 


\section{Statistical analysis}

Statistical analysis was done using $R$ Studio (1.3.959 using R version 4.0.0) with the following packages for data handling and statistics (tidyr, eeptools, reshape, dplyr, lubridate, phyloseq, vegan, microbiome, VIM, margrittr, chron, kableExtra, knitr, Isr, reshape2, multilevel, randomForest, factoextra, cluster, fpc, mice, miceadds, Ime4, nlme, data.table, stringr) and plotting (corrplot, ggplot2, ggpubr, lattice, ggfortify, sjPlot, cowplot, plotly, RColorBrewer, gridGraphics) [82-116].

We ran linear models to examine alpha diversity changes with age while controlling for batch ( 5 different runs) and breastfeeding ( 0 for never or rarely breastfed, 1 for occasionally, regularly, or daily breastfeeding). To examine the batch effect on gut bacterial diversity, we also ran a one-way ANOVA to estimate h2 for batch versus age. With an Adonis test (a randomization/Monte Carlo permutation test), we examined age-related changes in beta diversity (differences between infants). To test whether a sleep-gut link exists in infants, we applied multilevel models with the three markers for gut bacterial profiles (gut bacterial diversity, bacterial maturity index, and enterotype) as dependent variables and the five sleep composites (Sleep Day, Sleep Night, Sleep Timing, Sleep Activity, and Sleep Variability) as independent variables. Random intercepts were set for each participant. Due to the differences mentioned above, we controlled exact age, sex, batch, and breastfeeding.

Additionally, generalized linear models were computed for each assessment time point at 3, 6, and 12 months. Where enterotype was the outcome, generalized (multilevel) models with a binomial distribution were applied. Further, we examined the influence of enterotype pattern on sleep behavior at 12 months by using five generalized linear models. Each had one sleep composites at 12 months as the outcome and the enterotype evolution patterns as predictor variables (compared to the most common enterotype pattern, "Switcher").

The five sleep composites and the three gut bacteria markers were compared to behavioral development at 3, 6, and 12 months to examine the sleep-gut-brain link. For predictive associations, behavioral outcomes at 6, 12, and 24 months were included. For predictive associations, behavioral outcomes at 6, 12, and 24 months were included. We used random intercept cross-lagged panel models [117] to separate within-person and between-person variance in longitudinal analyses by 
including a latent intercept for each construct across assessment time points. The three behavioral outcomes (Collective Score, Gross Motor Score, and Personal

Social Score) were tested in a model including all five sleep composites and a model including all three gut bacterial markers, resulting in 6 models in total. We controlled for exact age and sex (at the intercept level) and breastfeeding for those models with bacterial predictors. These models did not consist of imputed data but instead estimated missing values using full information maximum likelihood (FIML). FIML is a general estimate of missing predictor data, yet not of outcome. We specified the five sleep composites as latent variables using the 32 sleep variables on which the composites are based [4]. For alpha diversity, we specified a latent variable onto which the three different diversity index load. Enterotypes, bacterial maturity index and the 3 behavioral outcomes were analyzed as manifest variables.

The alpha level was set to $P<0.05$. Results reaching significance only at trend level and results from the more restrictive version of the same dataset (outliers removed) are provided in the supplementary file.

\section{RESULTS}

\section{Characterization of gut bacteria across infancy}

Gut bacterial composition in infancy

Before addressing the primary investigation of the sleep-gut-brain link with the three gut bacterial markers, we started by characterizing the general maturation of gut bacterial profiles (Figure 2). First, we examined the abundance and prevalence of the core bacterial genera (Figure 2A). Bifidobacterium and Bacteroides were the most abundant genera across the first year of life. Infants maintained a similar overall composition of gut bacteria from 3 to 6 months of age, but a larger shift unfolded from 6 to 12 months.

\section{Gut bacterial diversity}

As expected, growing diversification with older age was also represented by the increase of alpha diversity (age effect: Shannon $\mathrm{t}(436)=11.74, \mathrm{~b}=0.13$ per month older, Observed $\mathrm{t}(436)=20.19, \mathrm{~b}=13.09$, Chao1 $\mathrm{t}(436)=17.46, \mathrm{~b}=14.49$, all $\mathrm{p}<$ 0.001 ; Figure 2B). Batch affected diversity measures (specifically the 4 th run), yet, however, explained proportionally little variance in comparison to the effect of age (Shannon $\mathrm{t}(436)=-4.24, \mathrm{~b}=-0.31$, Observed $\mathrm{t}(436)=-3.48, \mathrm{~b}=-18.54$, Chao 1 
$\mathrm{t}(436)=-3.86, \mathrm{~b}=-23.68$, all $\mathrm{p}<0.001$; effect size eta-squared $1.4 \%$ batch vs $26.6 \%$ age). An effect of breastfeeding was observed in Shannon only, such that predominantly breastfed infants had reduced gut bacterial diversity $(\mathrm{t}(436)=-2.04, \mathrm{~b}$ $=-0.04, p=0.04)$.

With Bray Curtis distance, we estimated beta diversity to quantify the dissimilarity between infants, which was significantly driven by age (Adonis, $F(3,449)=19.97, p=$ $0.001, \mathrm{R} 2=0.12$ ). While there was a substantial overlap at 3 and 6 months, there was a slight shift of the centroid, indicating some changes to the gut bacteria composition (ellipses on Figure 2C). Beta diversity at 12 months showed a notable shift in centroid compared and increasingly less overlap with beta diversity at 3 and 6 months.

\section{Bacterial Maturation Index}

The random forest model classified infants based on gut bacterial profiles to their age with $83.9 \%$ accuracy. Of particular interest were those bacterial profiles that deviated from correct age predictions: the model misclassified $14.5 \%$ of infants at 3 months (thereof 20 infants assigned to the older age 6 months, and 2 infants to even 12 months), $25.9 \%$ of infants at 6 months (thereof 33 infants assigned to younger age 3 months, yet 9 infants to 12 months), and $7.2 \%$ of infants at 12 months (1 infant assigned to 3 months, and 10 infants assigned to 6 months). The 20 top ranking age discriminatory bacterial OTUs comprised exclusively Firmicutes, 17 from Clostridiales order (6 from the Lachnospiraceae family), and 2 from Lactobacillales order, and 1 from an unknown order. 
A)
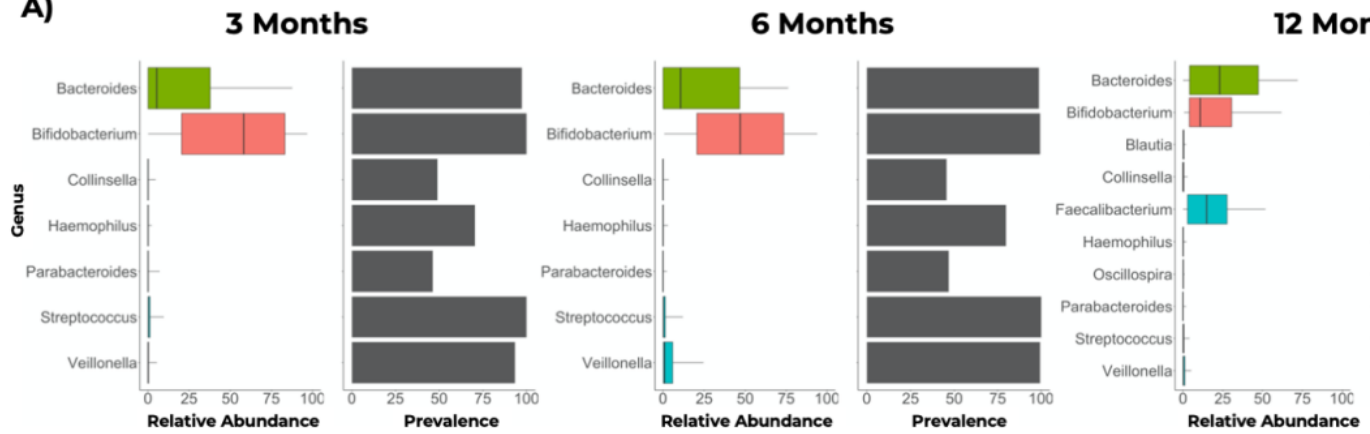

12 Months

B)

C)
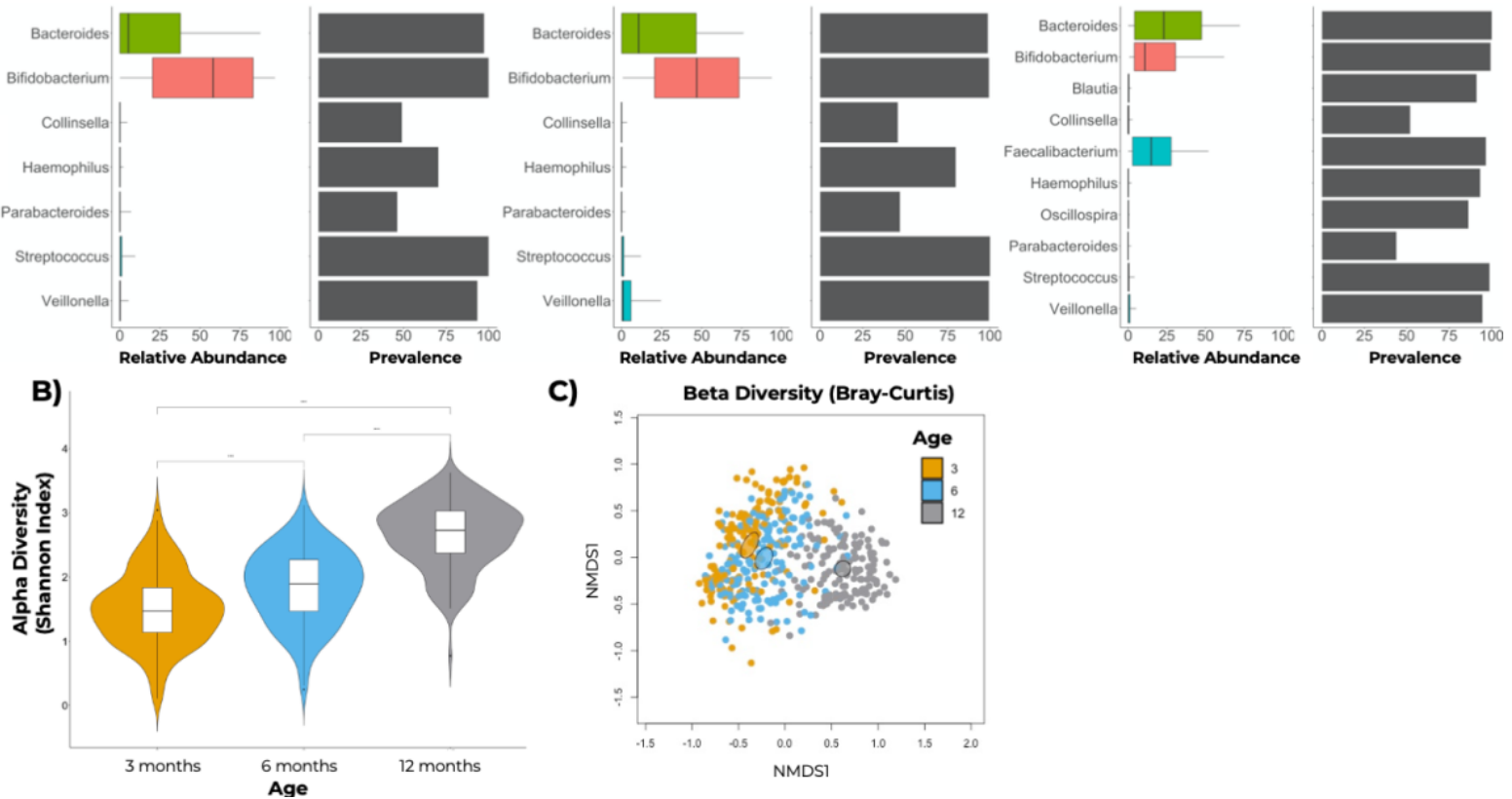

Relative Abundance

Prevalence

Figure 2. Maturational dynamics of infant gut bacteria across the first year of life. A) Prevalence and abundance (\%) of core bacterial genera at 3, 6, and 12 months of age. B) Significant increase of Shannon index with age, with $p<0.001$ among all alpha diversity measures: Observed, Shannon, and Chao1. C) Bray-Curtis based beta diversity to illustrate the age groups. 3 and 6 months of age highly overlap, with a close location of the centroids (ellipsis depicting 99\% Cl). The centroid for age 12 months is more notably distant to the younger groups, however, an overlap remains.

\section{Enterotype}

In the next step, we determined the representation of bacterial profiles as enterotypes in the sample. Weighted unifrac was performed across all ages with the clustering algorithm of Partitioning Around Medoids and identified the best fit in a 2cluster solution of 2 distinct enterotypes ( $A$ and $B$ ) with each representative bacterial composition. The two identified enterotypes differed primarily in the abundance of Bifidobacterium and Bacteroides. While enterotype A was primarily characterized by highly abundant Bifidobacterium and a low abundance of Bacteroides, enterotype $B$ demonstrated the reverse pattern. Enterotype $B$ additionally maintained more core genera, including some Firmicutes (Figure 3).

The number of infants assigned to each enterotype significantly changed across age. Specifically, more infants were assigned to enterotype $A$ at 3 and 6 months $(69.8 \%$ 
and $65.2 \%)$, whereas to enterotype B at 12 months of age $\left(83.6 \%, \chi^{2}(3)=107.46, p<\right.$ 0.001).

The developmental enterotype evolution patterns uncovered that approximately half of the infant population switched from enterotype $A$ to enterotype $B$ in the transition from 6 to 12 months (54.0\% "Switchers"). Nearly a third of infants consistently maintained enterotype B (29.0 \% "Bacteroides"), and a minority of infants remained with enterotype A (12.5\% "Bifidobacterium"). A small fraction of the infant population converted from B to A (4.6\% "Reverser").
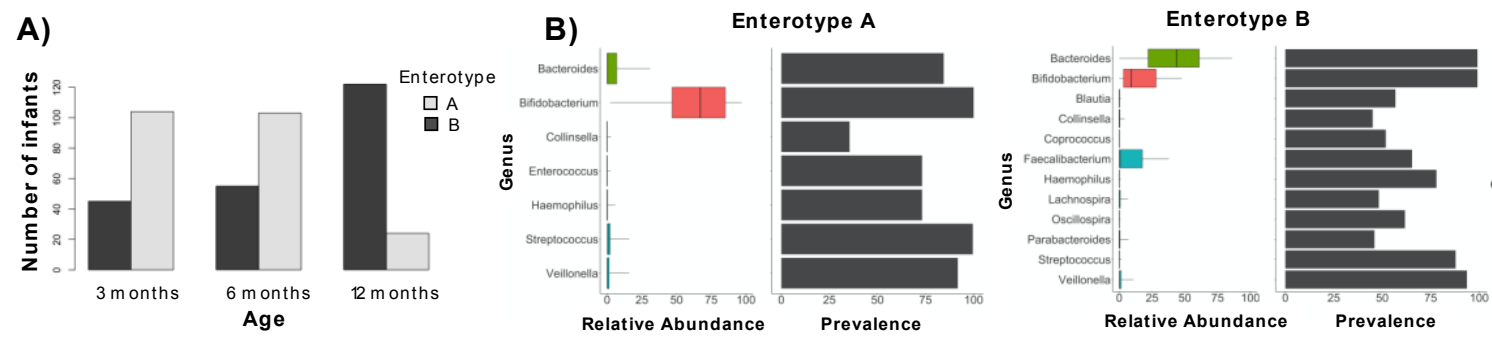

Figure 3. Weighted unifrac Partitioning Around Medoids analysis reveals two distinct enterotypes of gut bacteria composition in infancy. A) Number of infant stool samples assigned to enterotype A or B at each age based on a clustering approach. B) Characterization of the two infant enterotypes through abundance and prevalence of bacterial genera. Enterotype $A$ is characterized by a high abundance and prevalence of Bifidobacterium, while enterotype $B$ is characterized by the highest abundance of Bacteroides.

\section{Investigation of the sleep-gut link}

Extent of infant daytime sleep is associated with gut bacterial diversity

We then examined whether and how infant sleep patterns relate to gut bacterial diversity. We computed three multilevel models across each age, with alpha diversity as the outcome and sleep composites as independent variables (controlling for age, breastfeeding, sex, batch). We identified a negative association between Sleep Day and alpha diversity: infants with more diverse bacterial profiles generally demonstrated less predominant daytime sleep, thereby suggesting more "advanced" 
patterns in both domains even when controlling for age (Figure 4). The effect reached significance in Observed $\left(\mathrm{t}_{(240.90)}=-2.14, \mathrm{p}=0.03\right)$ and Chao1 $\left(\mathrm{t}_{(332.09)}=\right.$ 2.29, $\mathrm{p}=0.02)$ and a trend in Shannon $\left(\mathrm{t}_{(392.95)}=-1.75, \mathrm{p}=0.08\right)$. The other core infant sleep domains Sleep Night, Sleep Timing, Sleep Activity, and Sleep Variability were not significantly associated with gut bacterial diversity $(p>0.1$, Table 1$)$. To examine if this association is dynamic across the first year of life, we separated linear models for each assessment timepoint. At 3 months alpha diversity was strongly linked to Sleep Day (Observed $\mathrm{t}_{(83.66)}=-2.95, \mathrm{p}=0.004$, Chao1 $\mathrm{t}_{(98.66)}=$ 2.73, $p=0.008$, Shannon $\left.\mathrm{t}_{(105.54)}=-2.11, \mathrm{p}=0.04\right)$, which was not the case at older ages (all $p>0.1)$.

A)

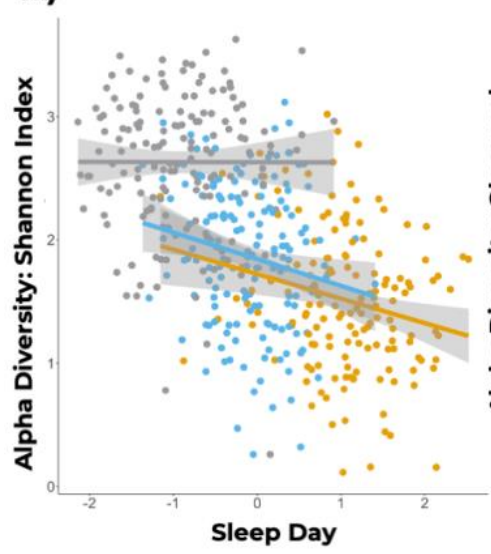

B)

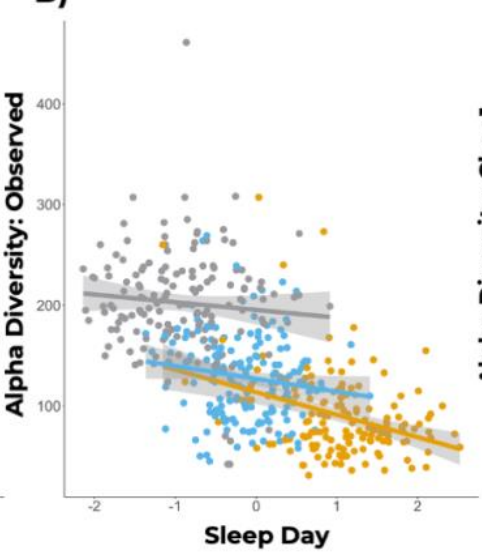

C)

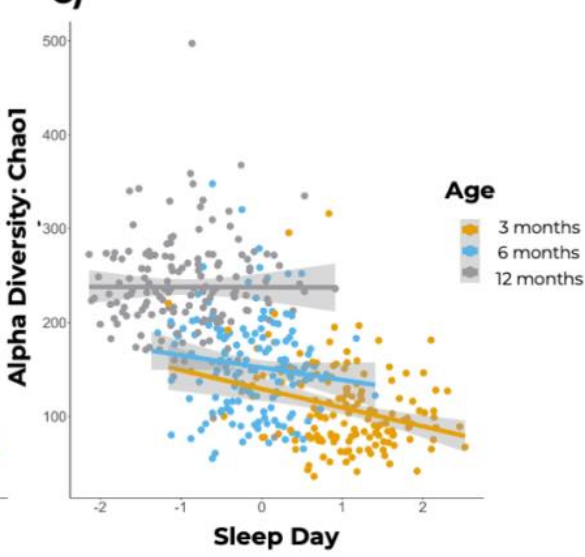

Figure 4. One representative imputation demonstrates the association between alpha diversity and Sleep Day, the primary infant sleep composite characterizing duration and number of daytime naps and their regularity. The finding shows that infants with more daytime sleep have lower alpha diversity. A) Shannon Diversity was significantly associated with Sleep Day at 3 months $(p=0.04)$, yet not at other ages. B) Observed diversity was significantly associated with Sleep Day across all ages (multilevel model, $p=0.03$ ) and at 3 months $(p=0.004)$, but not at other ages.

C) Chao diversity was significantly associated with Sleep Day across all ages (multilevel model, $p=0.02$ ) and at 3 months $(p=0.008)$, but not at other ages.

Infants with more mature bacteria wake up more at nighttime 
We then used the bacterial maturation index to examine whether infants classified as older/younger than their effective age according to their gut bacteria would also show a more mature/less mature sleep pattern. We computed a multilevel model with the bacterial maturation index as the outcome, all sleep composites as predictors, while controlling for exact age, sex, breastfeeding, and batch. Indeed, Sleep Activity was significantly linked to the bacterial maturation index $\left(t_{(348.91)}=2.23, p=0.03\right.$, Table 2 ), indicating that infants with more mature gut bacterial profiles also showed more activity and awakenings during sleep. This effect was evident only when all infant ages were combined and disappeared when age time points were considered separately (all sleep composites $p>0.05$ ).

\section{Enterotype}

Lastly, we investigated if sleep differs between infants assigned to enterotype A vs. enterotype B. No significant group differences in sleep composites were found neither across all time points nor within assessment time points (independent variables sleep composites, controlling for exact age, sex, and breastfeeding; all $p>$ 0.05, Table 3).

When comparing the enterotype evolution patterns ("Switchers", "Bifidobacterium", "Bacteroides" and "Reverser") infants age 12 months with the "Bifidobacterium" pattern had significantly increased Sleep Activity compared to infants in the "Switcher" pattern $\left(\mathrm{t}_{(120.43)}=2.00, \mathrm{p}=0.048\right)$. Additionally, infants with "Bacteroides" evolution pattern showed higher Sleep Variability compared to infants in the "Switcher" pattern $\left(\mathrm{t}_{(130.84)}=2.27, \mathrm{p}=0.02\right)$.

\section{Sleep and gut bacteria as predictors for developmental behavioral outcomes}

We examined whether infant sleep behavior and gut bacterial composition, 1) relate to their concurrent behavioral abilities, and 2) whether they predict later infant behavioral skills. We ran six random-intercept cross-lagged panel models, two for each behavioral outcome (Collective Score, Gross Motor Score, Personal Social Score), one with all sleep composites, the other with the three gut bacterial markers. We present each predictor variable (sleep behavior, gut bacterial markers) separately and only report significant findings in the text (for $b \pm$ SE see Figure 6). 


\section{Sleep Day}

Analyses for concurrent behavior at 3 months revealed a negative link between Sleep Day and behavioral skills, including Collective $(z=-2.08, p=0.04)$, Gross Motor ( $z=-2.46, p=0.01)$ and Personal Social scores $(z=2.00, p=0.045)$. Analyses for predictive behavior revealed a negative prediction of Gross Motor score at 24 months by Sleep Day at 12 months $(z=-2.09, p=0.04)$. Thus, infant daytime sleep patterns are linked to behavior - concurrently at 3 months for multiple behavioral domains and predictively for later motor skills at age 2 years.

\section{Sleep Night}

Analysis at 3 months showed that Sleep Night was positively associated with the concurrent Personal Social Score $(z=2.80, p=0.005)$. At 12 months Sleep Night was positively associated with concurrent Gross Motor Score $(z=2.09, p=0.04)$. Therefore, infants with more nighttime sleep revealed increased concurrent behavioral performance at 3 and 12 months.

\section{Sleep Activity}

At 3 months, Sleep Activity was positively linked with the concurrent Personal Social behavior $(z=2.00, p=0.045)$, indicating that 3 months-old infants with more activity and awakenings during the night have increased developmental performance.

\section{Sleep Timing}

Sleep Timing at 6 months negatively predicted the Personal social Score at 12 months $(z=-2.56, p=0.008)$. Thus, infants with later sleep times at age 6 months had lower behavioral developmental scores at 12 months.

\section{Sleep Variability}

Analyses showed that at 6 months Sleep Variability was positively associated with the concurrent Gross Motor Score $(z=2.44, p=0.02)$. Further, Sleep Variability at 6 months positively predicted the Collective Score at 12 months $(z=2.05, p=0.04)$. Overall, infants with more variable sleep patterns had increased behavioral developmental scores - both concurrently and predictively. 
Gut bacteria and behavioral development

Gut Bacterial Diversity

Analyses at infant age 3 months revealed that the gut bacterial diversity was positively associated with the concurrent Gross Motor Score $(z=2.15, p=0.03)$. At 12 months, alpha diversity was positively associated with the concurrent Collective Score $(z=2.00, p=0.045)$ and Gross Motor Score $(z=2.10, p=0.04)$. Therefore, infants with higher alpha diversity performed better in concurrent behavioral assessments.

Bacterial Maturity Index

Analyses showed that the bacterial maturity index at infant age 3 months was positively associated with the concurrent Gross Motor score $(z=2.69, p=0.007)$. Therefore, infants with more mature gut bacteria at 3 months scored higher in motor behavior.

\section{Enterotype}

Predictive analyses showed that the enterotype $A$ at 6 months predicted higher Gross Motor Scores at 12 months $(z=2.12, p=0.03)$. 
Same Age

Lagged (+ 1 assessment timepoint)

\begin{tabular}{|c|c|c|c|c|c|c|}
\hline 3 Months & Collective Score & Gross Motor & Personal Social & Collective Score & Gross Motor & Personal Social \\
\hline \multicolumn{7}{|l|}{ Sleep Measures } \\
\hline \multirow{2}{*}{$\begin{array}{l}\text { Sleep Activity } \\
\text { Sleep Night }\end{array}$} & $0.15 \pm 0.14$ & $0.14 \pm 0.12$ & $0.21 \pm 0.10$ & $0.12 \pm 0.10$ & $0.14 \pm 0.12$ & $0.09 \pm 0.12$ \\
\hline & $0.10 \pm 0.16$ & $0.12 \pm 0.15$ & $0.26 \pm 0.09$ & $-0.004 \pm 0.8$ & $0.08 \pm 0.09$ & $0.10 \pm 0.08$ \\
\hline Sleep Day & $-0.10 \pm 0.05$ & $0.10 \pm 0.04$ & $-0.08 \pm 0.04$ & $-0.18 \pm 0.36$ & $0.45 \pm 0.41$ & $-0.42 \pm 0.37$ \\
\hline \multirow{2}{*}{$\begin{array}{l}\text { Sleep Timing } \\
\text { Sleep Variability }\end{array}$} & $-0.08 \pm 0.08$ & $-0.12 \pm 0.07$ & $-0.14 \pm 0.08$ & $0.05 \pm 0.11$ & $0.23 \pm 0.13$ & $-0.12 \pm 0.12$ \\
\hline & $0.009 \pm 0.13$ & $-0.18 \pm 0.11$ & $0.16 \pm 0.11$ & $0.16 \pm 0.09$ & $0.01 \pm 0.12$ & $0.03 \pm 0.10$ \\
\hline \multicolumn{7}{|l|}{ Gut bacteria Measures } \\
\hline \multirow{3}{*}{$\begin{array}{l}\text { Alpha diversity } \\
\text { Enterotype } \\
\text { Bacterial Maturation Index }\end{array}$} & $0.09 \pm 0.12$ & $0.16+0.08$ & $0.11 \pm 0.09$ & $0.07 \pm 0.13$ & $0.09 \pm 0.16$ & $0.14 \pm 0.13$ \\
\hline & $0.13 \pm 0.08$ & $0.07 \pm 0.06$ & $0.06 \pm 0.06$ & $0.20 \pm 0.13$ & $0.27 \pm 0.16$ & $-0.05 \pm 0.15$ \\
\hline & $0.23 \pm 0.16$ & $0.31 \pm 0.11$ & $-0.07 \pm 0.12$ & $-0.04 \pm 0.08$ & $0.13 \pm 0.10$ & $-0.06 \pm 0.09$ \\
\hline \multicolumn{7}{|l|}{6 Months } \\
\hline \multicolumn{7}{|l|}{ Sleep Measures } \\
\hline \multirow{2}{*}{$\begin{array}{l}\text { Sleep Activity } \\
\text { Sleep Night }\end{array}$} & $-0.03 \pm 0.13$ & $0.14 \pm 0.12$ & $-0.11 \pm 0.11$ & $0.10 \pm 0.10$ & $-0.02 \pm 0.13$ & $0.27 \pm 0.14$ \\
\hline & $-0.03 \pm 0.13$ & $-0.09 \pm 0.11$ & $0.04 \pm 0.09$ & $-0.05 \pm 0.09$ & $-0.08 \pm 0.12$ & $0.11 \pm 0.10$ \\
\hline \multirow{3}{*}{$\begin{array}{l}\text { Sleep Day } \\
\text { Sleep Timing } \\
\text { Sleep Variability }\end{array}$} & $0.01 \pm 0.04$ & $0.01 \pm 0.03$ & $-0.06 \pm 0.03$ & $0.11 \pm 0.39$ & $-0.30 \pm 0.45$ & $0.41 \pm 0.47$ \\
\hline & $-0.06 \pm 0.09$ & $0.09 \pm 0.08$ & $-0.12 \pm 0.08$ & $-0.17 \pm 0.10$ & $-0.09 \pm 0.12$ & $-0.31 \pm 0.12$ \\
\hline & $0.10 \pm 0.14$ & $0.29 \pm 0.12$ & $0.06 \pm 0.11$ & $0.19 \pm 0.09$ & $0.11 \pm 0.12$ & $0.16 \pm 0.12$ \\
\hline \multicolumn{7}{|l|}{ Gut bacteria Measures } \\
\hline \multirow{3}{*}{$\begin{array}{l}\text { Alpha diversity } \\
\text { Enterotype } \\
\text { Bacterial Maturation Index }\end{array}$} & $-0.17 \pm 0.20$ & $0.07 \pm 0.07$ & $0.07 \pm 0.08$ & $0.11 \pm 0.13$ & $0.15 \pm 0.16$ & $0.28 \pm 0.16$ \\
\hline & $0.09 \pm 0.09$ & $-0.02 \pm .05$ & $-0.05 \pm 0.05$ & $0.17 \pm 0.14$ & $0.33 \pm 0.15$ & $-0.08 \pm 0.17$ \\
\hline & $-0.50 \pm 0.34$ & $0.05 \pm 0.12$ & $0.06 \pm 0.12$ & $-0.13 \pm 0.09$ & $-0.03 \pm 0.10$ & $-0.13 \pm 0.11$ \\
\hline \multicolumn{7}{|l|}{12 Months } \\
\hline \multicolumn{7}{|l|}{ Sleep Measures } \\
\hline \multirow{5}{*}{$\begin{array}{l}\text { Sleep Activity } \\
\text { Sleep Night } \\
\text { Sleep Day } \\
\text { Sleep Timing } \\
\text { Sleep Variability }\end{array}$} & $-0.16 \pm 0.14$ & $-0.11 \pm 0.12$ & $-0.18 \pm 0.11$ & $-0.13 \pm 0.12$ & $-0.15 \pm 0.12$ & $-0.10 \pm 0.14$ \\
\hline & $0.03 \pm 0.12$ & $0.22 \pm 0.11$ & $-0.02 \pm 0.09$ & $-0.10 \pm 0.09$ & $0.03 \pm 0.09$ & $0.0004 \pm 0.09$ \\
\hline & $-0.03 \pm 0.03$ & $-0.008 \pm 0.03$ & $-0.03 \pm 0.03$ & $-0.42 \pm 0.41$ & $-0.98 \pm 0.47$ & $-0.78 \pm 0.49$ \\
\hline & $-0.05 \pm 0.08$ & $-0.006 \pm 0.07$ & $-0.06 \pm 0.07$ & $-0.09 \pm 0.12$ & $0.08 \pm 0.14$ & $-0.20 \pm 0.14$ \\
\hline & $-0.04 \pm 0.14$ & $-0.02 \pm 0.11$ & $-0.05 \pm 0.11$ & $-0.001 \pm 0.11$ & $0.003 \pm 0.13$ & $-0.06 \pm 0.12$ \\
\hline \multicolumn{7}{|l|}{ Gut bacteria Measures } \\
\hline \multirow{3}{*}{$\begin{array}{l}\text { Alpha diversity } \\
\text { Enterotype } \\
\text { Bacterial Maturation Index }\end{array}$} & $0.17 \pm 0.08$ & $0.16 \pm 0.08$ & $0.11 \pm 0.09$ & $0.15 \pm 0.12$ & $-0.11 \pm 0.16$ & $0.06 \pm 0.14$ \\
\hline & $-0.02 \pm 0.05$ & $-0.02 \pm 0.05$ & $-0.06 \pm 0.05$ & $-0.17 \pm 0.17$ & $-0.12 \pm 0.20$ & $-0.23 \pm 0.20$ \\
\hline & $0.03 \pm 0.12$ & $0.03 \pm 0.11$ & $-0.08 \pm 0.13$ & $0.05 \pm 0.07$ & $0.16 \pm 0.09$ & $-0.07 \pm 0.09$ \\
\hline
\end{tabular}

Figure 6. Associations between infant sleep composites, the three gut bacteria measures and behavioral skills at 3, 6 , and 12 months of age ( $\beta \pm$ Standard Error). Left columns contain statistics for concurrent associations (e.g., 3 months in relation to 3 months), right columns indicate associations lagged by one assessment timepoint (e.g., 3 months in relation to 6 months). Blue color denotes positive association, while red color denotes negative associations.

\section{DISCUSSION}

We examined whether a sleep-gut link exists across the first months of human life by means of a large longitudinal cohort of healthy infants. We quantified infant sleep behavior, gut bacterial markers, and behavioral developmental status at ages 3, 6, and 12 months. Indeed, our results overall confirm the existence of a sleep-gut link in infants. Specifically, the expression of infant daytime sleep was related to alpha diversity in stool samples, and nighttime awakenings were related to the bacterial 
maturity index as well as the enterotype. Further, the sleep-gut link was dynamic across the infant period, such that the specific sleep-gut associations changed across development. Finally, both - sleep behavior and gut bacteria were connected with infant behavioral developmental status, with the most prominent associations of sleep-behavior and gut-behavior at age 3 months.

\section{Maturation of Gut Bacteria}

\section{Composition and Diversity}

Gut bacteria composition and its maturation emerged in the patterns as expected for infants. We reported a marked increase in alpha diversity but a decrease in beta diversity across the first year of life, which agrees with previous findings $[118,119]$. As previously reported, Bifidobacterium was the most abundant genus in the first few months of life, while at 12 months, Bacteroides became increasingly abundant $[120,121]$. Furthermore, the observed increasing prevalence and abundance of Firmicutes at 12 months is in alignment with previous research [120].

\section{Enterotypes}

We provide new evidence that healthy infants can be assigned to two enterotypes based on their gut bacterial profiles. One enterotype $(A)$ is characterized by high abundance of Bifidobacterium; the other $(B)$ is represented by increased abundance of Bacteroides. In contrast, a study with neonates and young infants reported the existence of 3 enterotypes infants - one characterized by a high abundance of Proteobacteria, another by a high abundance of Actinobacteria, and a third with highly abundant Firmicutes [122]. Interpretations considered that these enterotypes differed geographically and that in Western societies, the Actinobacteria enterotype was the most common. The latter likely corresponds to enterotype $A$ in our sample (characterized by high Bifidobacterium). Relevant to this discussion is our reporting that most infants switch across their first year of life from the Bifidobacterium enterotype (A) to the Bacteroides enterotype (B). The enterotypes identified in our Swiss sample differ from the ones reported in an American sample in Carlson et al., 2018 [37], which identified 3 enterotypes at 12 months old. Namely, while we similarly observe an increase in abundance of Faecalibacterium at 12 months, our computation does yet not identify a clearly separable third cluster. Of note, our sample possibly reveals overall reduced gut bacterial diversity, due to our strict 
inclusion criteria (infants breastfed for 3 months and born naturally, i.e., no Csection), which were chosen to reduce interindividual variance of known influences, yet which may have influenced the enterotype characterization.

\section{Sleep}

\section{Sleep Day}

Our results demonstrate that infant daytime sleep (Sleep Day) is linked to infants' gut bacterial profile (alpha diversity): Infants who slept more during the day had lower diversity compared to infants who slept proportionally less during the day. The effect was most substantial at 3 months and gradually decreased thereafter. This finding is exciting because we recently identified Sleep Day also as the most relevant player linked to infant behavioral development among other comprehensively tested sleep behaviors [4]. Yet, why the sleep-gut association weakens across development is unclear. Potentially, this phenomenon identifies an early sensitive period for later functionality of sleep rhythm and gut balance. It is particularly the first year of life, that entails the most drastic changes of both daytime sleep and alpha diversity [123]. Further, daytime sleep gradually decreases across infancy into the preschool years [3]. It is thus possible that in the infant period, the build-up of sleep-profiles and gut bacterial profiles are connected, while in the later period of refinement of both profiles happens more distantly, and possible includes additional factors.

Daytime sleep reflects processes of human brain development. The observation that Sleep Day at 12 months predicts 24-month motor development is novel and adds further evidence to the growing concept of Sleep Day as core maturational marker $[4,124,125]$. In line with this, the amount of wakefulness at birth (i.e., inverse to our Sleep Day) was previously positively linked to motor development [126]. A comprehensive electrophysiological investigation of naps across early childhood further supports the concept that more daytime sleep suggest a less mature neural system, as evidenced by increased sleep pressure (slow wave activity) when nap are moved to later clock times of the day [125]. Additionally, young children who habitually nap were observed to have less mature cognitive networks, assessed as hippocampal volume, compared to children who have ceased napping [124]. 
In adults and rodents, increased alpha diversity has been associated with better sleep quality $[39,49]$. Sleep quality is traditionally measured by nighttime awakenings in older children or adults. However, we did not observe any associations between Sleep Activity - the composite that includes nighttime awakenings - and alpha diversity in our infant sample.

\section{Sleep Activity}

Nighttime awakenings are common in infants, especially at 3 and 6 months. At 6 months, consistently sleeping through for 8 hours is observed in less than $3 \%$ of all infants [127]. Surprisingly, our results demonstrate that Sleep Activity at 3 months is positively linked to personal social development. We observed a notable developmental transition in the association between Sleep Activity with developmental outcomes. On a descriptive level, albeit not significant, connections between Sleep Activity and behavioral development are positive, at 6 months associations mixed, whereas at 12 and 24 months negative. We thus propose a new model that Sleep Activity transitions from first being a "functional" behavior with the purpose to regularly wake up in order to being fed, to second becoming a behavior not anymore linked to survival. In the latter, Sleep Activity becomes "dysfunctional" and turns into an indicator for reduced sleep quality. Similarly, while toddlers seem to exhibit a linear relationship between nighttime awakenings and cognitive function, this association is not linear in infants. Interestingly, the association seems to be in a reverse u-shape in infants, so that infants in the midrange scored highest on the mental development index [128].

Potentially the maturational change in the functionality of Sleep Activity is related to research methodology. Sleep Activity in actigraphy is computed from movement, and thus quantifies a subject's motor activity which can also occur during sleep instead of uniquely the periods of wakefulness at nighttime. While we used data smoothing to only include periods of activity that are longer than 5 minutes, potentially more extended REM periods are still captured in this composite. This is particularly the case for infants with generally high levels of activity (also during sleep) [129]. Therefore, especially in the computations at earliest ages, wakefulness during the night might be overestimated and Sleep Activity might contain movement activity during sleep more so than effective nocturnal wakefulness. Motor activity during 
sleep in infants includes numerous twitches in REM sleep [130]. These twitches seem to trigger the neurophysiological maturation of the motor cortex, specifically by activating and thereby organizing sensorimotor networks [131,132].

We find an association between Sleep Activity and less mature enterotype patterns ("Bifidobacterium") at 12 months. Previously it has been reported that sleep disruptions decrease Bifidobacterium abundance [48]. However, it is of note that most infants assigned to enterotype $B$ nonetheless demonstrate increased relative abundance of Bifidobacterium than reported in adults [119]. Likely two different mechanisms underlie the observed dynamics in Bifidobacterium based on sleep disruption vs. awakenings in infants.

\section{Sleep Variability}

In adults, high variability in sleep behaviors has been linked with poorer academic performance [66]. Surprisingly, Sleep Variability was positively associated with behavioral outcomes at 6 and 12 months. As sleep becomes less variable across the first year of life, higher variability is a more immature sleep behavior. Higher variability could potentially be related to parents' higher attunement to the baby's cues and personal needs. Potentially, the more variable schedule could allow the infant to sleep when it is most needed (e.g., after learning something new [133]) rather than at pre-specified times.

\section{Sleep Night}

We did not find any association of Sleep Night with the three gut bacterial markers. Previous studies in adults have reported changes in gut bacteria profiles after partial sleep deprivation [47], yet another study did not find solid effects of sleep restriction [50]. However, infant sleep duration was not experimentally altered in the current investigation, which may explain the lack of effects.

Infant nighttime sleep duration was linked to behavioral outcomes, such that longer sleep duration was associated with increased personal social scores at 3 months and higher gross motor scores at 12 months. This extends existing knowledge to the infant domain, as sleep duration in toddlers was previously linked to cognitive performance [134]. 


\section{Sleep Timing}

We did not find any association of Sleep Timing with our three gut bacterial markers. Previous studies had reported that disrupted circadian rhythms influenced the gut bacteria composition $[42,44]$. However, while the infants in our study showed variability in the preferred Sleep Timing, none showed a strong disruption of circadian rhythms, which might explain a lack of effect. Furthermore, we focused on sleep behavior (e.g., bedtimes) rather than circadian patterns (e.g., inter-day stability).

We found limited evidence for an effect of Sleep Timing on behavioral outcomes. Late sleep timing at 6 months negatively predicted Personal Social development at 12 months. Potentially, this effect was only visible at 6 months, because most infants changed to earlier Sleep Timing from 3 to 6 months, and subsequently retained stable timing.

\section{Gut Bacteria Behavior Link}

\section{Alpha Diversity}

Behavioral development was positively linked to alpha diversity at 3 and 12 months. A negative association between alpha diversity at 12 months and development at 24 months has been previously reported [37], which we did not observe for these ages. Potentially, the different developmental behaviors investigated (visual reception, expressive language vs gross motor and personal social development) led to this divergence in results $[63,135]$.

\section{Enterotype}

Enterotype was generally only weakly connected to behavioral development, however, 6-month-olds with enterotype A had increased motor skills at age 12 months. As most infants switch from enterotype A to enterotype B between 6 and 12 months, this period potentially reflects a sensitive transition in bacterial profiles.

\section{Bacterial Maturity Index}

The Bacterial Maturity Index was only associated with early motor skills. Infants with more mature bacteria showed comparably advanced motor skills at 3 months. Immature bacterial profiles have previously been linked to malnourishment [36], but 
to our knwoledge no study has investigated the link of bacterial maturity to behavioral development.

\section{Limitations}

Our study uses a correlational design which does not allow for causal conclusions. To address the concerns voiced about cross-lagged panel models regarding the separation of the within-person and between-person trait like differences we used a random intercept cross-lagged panel model [117].

Due to the longitudinal data collection across 4 years, gut bacteria were analyzed across five batches. This created differences of sequencing depth between runs, which especially influenced alpha diversity. We counteracted this effect with two actions: only including samples exceeding 50'000 reads, and accounting for analysis run with a covariate whenever possible (exceptions were the random-intercept crosslagged panel models and the models using enterotype as a predictor).

Furthermore, reads were detected in our negative control samples (water probes). However, ZOTU2, which was the most common OTU in the negative control samples, only influenced the 3 gut bacteria markers to a negligible extent. Our findings might not generalize to infants born through c-section or who were bottle-fed in early infancy, as we purposely included natural births and breastfed infants to account for known effects in gut bacteria [120,121].

\section{Implications}

These findings are of great clinical relevance because both, sleep and gut bacteria, are modifiable. Sleep can be modified using healthy behavioral interventions through parents, e.g., education about infant sleep rhythm maturation and specific behavioral strategies to improve sleep $[20,136]$. Gut bacteria can be modified by nutrition or orally ingested pre- and probiotics added to infant formula $[33,137]$. Even though specific recommendations cannot be made with the current state of knowledge, there is large potential to alter the sleep-gut link from either direction.

\section{Summary}

This study is the first to show the existence of the sleep-gut link in infancy. Both sleep and gut bacteria undergo rapid maturational transitions, and these findings 
demonstrate dynamic connections between the two. Specifically, we found that infant daytime sleep is associated with alpha diversity and motor activity during sleep, and that nighttime waking is associated with both bacterial maturity and bacterial enterotype. Furthermore, both sleep and gut bacteria are related to behavioral development across the first year of life, with the strongest association at 3 months. Potentially, this phenomenon identifies an early sensitive period for later functionality of sleep rhythm and gut bacterial balance.

1. Boyle, C.A.; Boulet, S.; Schieve, L.A.; Cohen, R.A.; Blumberg, S.J.; Yeargin-Allsopp, M.; Visser, S.; Kogan, M.D. Trends in the Prevalence of Developmental Disabilities in US Children, 1997-2008. Pediatrics 2011, 127, 1034-1042, doi:10.1542/peds.2010-2989.

2. Shonkoff, J.P. Leveraging the Biology of Adversity to Address the Roots of Disparities in Health and Development. Proc. Natl. Acad. Sci. 2012, 109, 17302-17307, doi:10.1073/pnas.1121259109.

3. Iglowstein, I.; Jenni, O.G.; Molinari, L.; Largo, R.H. Sleep Duration From Infancy to Adolescence: Reference Values and Generational Trends. PEDIATRICS 2003, 111, 302307, doi:10.1542/peds.111.2.302.

4. Schoch, S.F.; Huber, R.; Kohler, M.; Kurth, S. Which Are the Central Aspects of Infant Sleep? The Dynamic of Sleep Composites across Infancy. bioRxiv 2020, 2020.10.26.354803, doi:10.1101/2020.10.26.354803.

5. Tononi, G.; Cirelli, C. Sleep Function and Synaptic Homeostasis. Sleep Med. Rev. 2006, 10, 49-62, doi:10.1016/j.smrv.2005.05.002.

6. Jha, S.K.; Jones, B.E.; Coleman, T.; Steinmetz, N.; Law, C.-T.; Griffin, G.; Hawk, J.; Dabbish, N.; Kalatsky, V.A.; Frank, M.G. Sleep-Dependent Plasticity Requires Cortical Activity. J. Neurosci. 2005, 25, 9266-9274, doi:10.1523/JNEUROSCI.2722-05.2005.

7. Jenni, O.G.; Borbély, A.A.; Achermann, P. Development of the Nocturnal Sleep Electroencephalogram in Human Infants. Am. J. Physiol.-Regul. Integr. Comp. Physiol. 2004, 286, R528-R538, doi:10.1152/ajpregu.00503.2003.

8. Tripathi, A.; Melnik, A.V.; Xue, J.; Poulsen, O.; Meehan, M.J.; Humphrey, G.; Jiang, L.; Ackermann, G.; McDonald, D.; Zhou, D.; et al. Intermittent Hypoxia and Hypercapnia, a Hallmark of Obstructive Sleep Apnea, Alters the Gut Microbiome and Metabolome. mSystems 2018, 3, doi:10.1128/mSystems.00020-18.

9. Louis, J.; Zhang, J.X.; Revol, M.; Debilly, G.; Challamel, M.J. Ontogenesis of Nocturnal Organization of Sleep Spindles: A Longitudinal Study during the First 6 Months of Life. Electroencephalogr. Clin. Neurophysiol. 1992, 83, 289-296, doi:10.1016/00134694(92)90088-Y.

10. Fagioli, I.; Salzarulo, P. Sleep States Development in the First Year of Life Assessed through 24-h Recordings. Early Hum. Dev. 1982, 6, 215-228, doi:10.1016/03783782(82)90109-8.

11. Fattinger, S.; Jenni, O.G.; Schmitt, B.; Achermann, P.; Huber, R. Overnight Changes in the Slope of Sleep Slow Waves during Infancy. Sleep 2014, 37, 245-253.

12. Schoch, S.F.; Riedner, B.A.; Deoni, S.C.; Huber, R.; LeBourgeois, M.K.; Kurth, S. AcrossNight Dynamics in Traveling Sleep Slow Waves throughout Childhood. Sleep 2018, 41, doi:10.1093/sleep/zsy165. 
13. Kurth, S.; Ringli, M.; Geiger, A.; LeBourgeois, M.; Jenni, O.G.; Huber, R. Mapping of Cortical Activity in the First Two Decades of Life: A High-Density Sleep

Electroencephalogram Study. J. Neurosci. 2010, 30, 13211-13219.

14. Kurth, S.; Achermann, P.; Rusterholz, T.; LeBourgeois, M.K. Development of Brain EEG Connectivity across Early Childhood: Does Sleep Play a Role? Brain Sci. 2013, 3, 14451460, doi:10.3390/brainsci3041445.

15. Tarokh, L.; Carskadon, M.A.; Achermann, P. Developmental Changes in Brain Connectivity Assessed Using the Sleep EEG. Neuroscience 2010, 171, 622-634, doi:10.1016/j.neuroscience.2010.08.071.

16. Lam, P.; Hiscock, H.; Wake, M. Outcomes of Infant Sleep Problems: A Longitudinal Study of Sleep, Behavior, and Maternal Well-Being. Pediatrics 2003, 111, e203-e207, doi:10.1542/peds.111.3.e203.

17. Gregory, A.M.; Caspi, A.; Eley, T.C.; Moffitt, T.E.; O'Connor, T.G.; Poulton, R. Prospective Longitudinal Associations Between Persistent Sleep Problems in Childhood and Anxiety and Depression Disorders in Adulthood. J. Abnorm. Child Psychol. 2005, 33, 157-163, doi:10.1007/s10802-005-1824-0.

18. Taveras, E.M.; Rifas-Shiman, S.L.; Oken, E.; Gunderson, E.P.; Gillman, M.W. Short Sleep Duration in Infancy and Risk of Childhood Overweight. Arch. Pediatr. Adolesc. Med. 2008, 162, 305-311, doi:10.1001/archpedi.162.4.305.

19. Simola, P.; Liukkonen, K.; Pitkäranta, A.; Pirinen, T.; Aronen, E.T. Psychosocial and Somatic Outcomes of Sleep Problems in Children: A 4-Year Follow-up Study. Child Care Health Dev. 2014, 40, 60-67, doi:10.1111/j.1365-2214.2012.01412.x.

20. Price, A.M.H.; Wake, M.; Ukoumunne, O.C.; Hiscock, H. Five-Year Follow-up of Harms and Benefits of Behavioral Infant Sleep Intervention: Randomized Trial. Pediatrics 2012, 130, 643-651, doi:10.1542/peds.2011-3467.

21. Wake, M.; Price, A.; Clifford, S.; Ukoumunne, O.C.; Hiscock, H. Does an Intervention That Improves Infant Sleep Also Improve Overweight at Age 6? Follow-up of a Randomised Trial. Arch. Dis. Child. 2011, 96, 526-532, doi:10.1136/adc.2010.196832.

22. Hassan, F.; Davis, M.M.; Chervin, R.D. No Independent Association between Insufficient Sleep and Childhood Obesity in the National Survey of Children's Health. J. Clin. Sleep Med. 2011, 07, 153-157, doi:10.5664/jcsm. 28102.

23. Timofeev, I.; Schoch, S.F.; LeBourgeois, M.K.; Huber, R.; Riedner, B.A.; Kurth, S. SpatioTemporal Properties of Sleep Slow Waves and Implications for Development. Curr. Opin. Physiol. 2020, 15, 172-182, doi:10.1016/j.cophys.2020.01.007.

24. Huber, R.; Felice Ghilardi, M.; Massimini, M.; Tononi, G. Local Sleep and Learning. Nature 2004, 430, 78-81, doi:10.1038/nature02663.

25. Fattinger, S.; de Beukelaar, T.T.; Ruddy, K.L.; Volk, C.; Heyse, N.C.; Herbst, J.A.; Hahnloser, R.H.R.; Wenderoth, N.; Huber, R. Deep Sleep Maintains Learning Efficiency of the Human Brain. Nat. Commun. 2017, 8, 15405, doi:10.1038/ncomms15405.

26. Cryan, J.F.; O’Riordan, K.J.; Cowan, C.S.M.; Sandhu, K.V.; Bastiaanssen, T.F.S.; Boehme, M.; Codagnone, M.G.; Cussotto, S.; Fulling, C.; Golubeva, A.V.; et al. The MicrobiotaGut-Brain Axis. Physiol. Rev. 2019, 99, 1877-2013, doi:10.1152/physrev.00018.2018.

27. Hegstrand, L.R.; Hine, R.J. Variations of Brain Histamine Levels in Germ-Free and Nephrectomized Rats. Neurochem. Res. 1986, 11, 185-191, doi:10.1007/BF00967967.

28. Clarke, G.; Grenham, S.; Scully, P.; Fitzgerald, P.; Moloney, R.D.; Shanahan, F.; Dinan, T.G.; Cryan, J.F. The Microbiome-Gut-Brain Axis during Early Life Regulates the 
Hippocampal Serotonergic System in a Sex-Dependent Manner. Mol. Psychiatry 2013, 18, 666-673.

29. Gareau, M.G.; Wine, E.; Rodrigues, D.M.; Cho, J.H.; Whary, M.T.; Philpott, D.J.; MacQueen, G.; Sherman, P.M. Bacterial Infection Causes Stress-Induced Memory Dysfunction in Mice. Gut 2011, 60, 307-317, doi:10.1136/gut.2009.202515.

30. O’Mahony, S.M.; Felice, V.D.; Nally, K.; Savignac, H.M.; Claesson, M.J.; Scully, P.; Woznicki, J.; Hyland, N.P.; Shanahan, F.; Quigley, E.M. Disturbance of the Gut Microbiota in Early-Life Selectively Affects Visceral Pain in Adulthood without Impacting Cognitive or Anxiety-Related Behaviors in Male Rats. Neuroscience 2014, 277, 885-901.

31. Pinto-Sanchez, M.I.; Hall, G.B.; Ghajar, K.; Nardelli, A.; Bolino, C.; Lau, J.T.; Martin, F.P.; Cominetti, O.; Welsh, C.; Rieder, A.; et al. Probiotic Bifidobacterium Longum NCC3001 Reduces Depression Scores and Alters Brain Activity: A Pilot Study in Patients With Irritable Bowel Syndrome. Gastroenterology 2017, 153, 448-459.e8, doi:10.1053/j.gastro.2017.05.003.

32. Allen, A.P.; Hutch, W.; Borre, Y.E.; Kennedy, P.J.; Temko, A.; Boylan, G.; Murphy, E.; Cryan, J.F.; Dinan, T.G.; Clarke, G. Bifidobacterium Longum 1714 as a Translational Psychobiotic: Modulation of Stress, Electrophysiology and Neurocognition in Healthy Volunteers. Transl. Psychiatry 2016, 6, e939-e939, doi:10.1038/tp.2016.191.

33. Tillisch, K.; Labus, J.; Kilpatrick, L.; Jiang, Z.; Stains, J.; Ebrat, B.; Guyonnet, D.; LegrainRaspaud, S.; Trotin, B.; Naliboff, B.; et al. Consumption of Fermented Milk Product With Probiotic Modulates Brain Activity. Gastroenterology 2013, 144, 1394-1401.e4, doi:10.1053/j.gastro.2013.02.043.

34. Sudo, N.; Chida, Y.; Aiba, Y.; Sonoda, J.; Oyama, N.; Yu, X.-N.; Kubo, C.; Koga, Y. Postnatal Microbial Colonization Programs the Hypothalamic-Pituitary-Adrenal System for Stress Response in Mice. J. Physiol. 2004, 558, 263-275, doi:10.1113/jphysiol.2004.063388.

35. Heijtz, R.D.; Wang, S.; Anuar, F.; Qian, Y.; Björkholm, B.; Samuelsson, A.; Hibberd, M.L.; Forssberg, H.; Pettersson, S. Normal Gut Microbiota Modulates Brain Development and Behavior. Proc. Natl. Acad. Sci. 2011, 108, 3047-3052, doi:10.1073/pnas.1010529108.

36. Blanton, L.V.; Charbonneau, M.R.; Salih, T.; Barratt, M.J.; Venkatesh, S.; Ilkaveya, O.; Subramanian, S.; Manary, M.J.; Trehan, I.; Jorgensen, J.M.; et al. Gut Bacteria That Prevent Growth Impairments Transmitted by Microbiota from Malnourished Children. Science 2016, 351, doi:10.1126/science.aad3311.

37. Carlson, A.L.; Xia, K.; Azcarate-Peril, M.A.; Goldman, B.D.; Ahn, M.; Styner, M.A.; Thompson, A.L.; Geng, X.; Gilmore, J.H.; Knickmeyer, R.C. Infant Gut Microbiome Associated With Cognitive Development. Biol. Psychiatry 2018, 83, 148-159, doi:10.1016/j.biopsych.2017.06.021.

38. González-Mercado, V.J.; Sarkar, A.; Penedo, F.J.; Pérez-Santiago, J.; McMillan, S.; Marrero, S.J.; Marrero-Falcón, M.A.; Munro, C.L. Gut Microbiota Perturbation Is Associated with Acute Sleep Disturbance among Rectal Cancer Patients. J. Sleep Res. 2020, 29, e12915, doi:10.1111/jsr.12915.

39. Grosicki, G.J.; Riemann, B.L.; Flatt, A.A.; Valentino, T.; Lustgarten, M.S. Self-Reported Sleep Quality Is Associated with Gut Microbiome Composition in Young, Healthy Individuals: A Pilot Study. Sleep Med. 2020, 73, 76-81, doi:10.1016/j.sleep.2020.04.013. 
40. Smith, R.P.; Easson, C.; Lyle, S.M.; Kapoor, R.; Donnelly, C.P.; Davidson, E.J.; Parikh, E.; Lopez, J.V.; Tartar, J.L. Gut Microbiome Diversity Is Associated with Sleep Physiology in Humans. PLOS ONE 2019, 14, e0222394, doi:10.1371/journal.pone.0222394.

41. Poroyko, V.A.; Carreras, A.; Khalyfa, A.; Khalyfa, A.A.; Leone, V.; Peris, E.; Almendros, I.; Gileles-Hillel, A.; Qiao, Z.; Hubert, N.; et al. Chronic Sleep Disruption Alters Gut Microbiota, Induces Systemic and Adipose Tissue Inflammation and Insulin Resistance in Mice. Sci. Rep. 2016, 6, 35405, doi:10.1038/srep35405.

42. Voigt, R.M.; Forsyth, C.B.; Green, S.J.; Mutlu, E.; Engen, P.; Vitaterna, M.H.; Turek, F.W.; Keshavarzian, A. Circadian Disorganization Alters Intestinal Microbiota. PLOS ONE 2014, 9, e97500, doi:10.1371/journal.pone.0097500.

43. Voigt, R.M.; Summa, K.C.; Forsyth, C.B.; Green, S.J.; Engen, P.; Naqib, A.; Vitaterna, M.H.; Turek, F.W.; Keshavarzian, A. The Circadian Clock Mutation Promotes Intestinal Dysbiosis. Alcohol. Clin. Exp. Res. 2016, 40, 335-347, doi:10.1111/acer.12943.

44. Deaver, J.A.; Eum, S.Y.; Toborek, M. Circadian Disruption Changes Gut Microbiome Taxa and Functional Gene Composition. Front. Microbiol. 2018, 9, doi:10.3389/fmicb.2018.00737.

45. Thaiss, C.A.; Zeevi, D.; Levy, M.; Zilberman-Schapira, G.; Suez, J.; Tengeler, A.C.; Abramson, L.; Katz, M.N.; Korem, T.; Zmora, N.; et al. Transkingdom Control of Microbiota Diurnal Oscillations Promotes Metabolic Homeostasis. Cell 2014, 159, 514-529, doi:10.1016/j.cell.2014.09.048.

46. Liu, Z.; Wei, Z.-Y.; Chen, J.; Chen, K.; Mao, X.; Liu, Q.; Sun, Y.; Zhang, Z.; Zhang, Y.; Dan, Z.; et al. Acute Sleep-Wake Cycle Shift Results in Community Alteration of Human Gut Microbiome. mSphere 2020, 5, doi:10.1128/mSphere.00914-19.

47. Benedict, C.; Vogel, H.; Jonas, W.; Woting, A.; Blaut, M.; Schürmann, A.; Cedernaes, J. Gut Microbiota and Glucometabolic Alterations in Response to Recurrent Partial Sleep Deprivation in Normal-Weight Young Individuals. Mol. Metab. 2016, 5, 11751186, doi:10.1016/j.molmet.2016.10.003.

48. Bowers, S.J.; Vargas, F.; González, A.; He, S.; Jiang, P.; Dorrestein, P.C.; Knight, R.; Jr, K.P.W.; Lowry, C.A.; Fleshner, M.; et al. Repeated Sleep Disruption in Mice Leads to Persistent Shifts in the Fecal Microbiome and Metabolome. PLOS ONE 2020, 15, e0229001, doi:10.1371/journal.pone.0229001.

49. Maki, K.A.; Burke, L.A.; Calik, M.W.; Watanabe-Chailland, M.; Sweeney, D.; RomickRosendale, L.E.; Green, S.J.; Fink, A.M. Sleep Fragmentation Increases Blood Pressure and Is Associated with Alterations in the Gut Microbiome and Fecal Metabolome in Rats. Physiol. Genomics 2020, 52, 280-292, doi:10.1152/physiolgenomics.00039.2020.

50. Zhang, S.L.; Bai, L.; Goel, N.; Bailey, A.; Jang, C.J.; Bushman, F.D.; Meerlo, P.; Dinges, D.F.; Sehgal, A. Human and Rat Gut Microbiome Composition Is Maintained Following Sleep Restriction. Proc. Natl. Acad. Sci. 2017, 114, E1564-E1571, doi:10.1073/pnas.1620673114.

51. Brown, R.; Price, R.J.; King, M.G.; Husband, A.J. Are Antibiotic Effects on Sleep Behavior in the Rat Due to Modulation of Gut Bacteria? Physiol. Behav. 1990, 48, 561-565.

52. Jenni, O.G.; Carskadon, M.A. Spectral Analysis of the Sleep Electroencephalogram During Adolescence. Sleep 2004, 27, 774-783, doi:10.1093/sleep/27.4.774. 
53. Paulose, J.K.; Wright, J.M.; Patel, A.G.; Cassone, V.M. Human Gut Bacteria Are Sensitive to Melatonin and Express Endogenous Circadian Rhythmicity. PLOS ONE 2016, 11, e0146643, doi:10.1371/journal.pone.0146643.

54. Leone, V.; Gibbons, S.M.; Martinez, K.; Hutchison, A.L.; Huang, E.Y.; Cham, C.M.; Pierre, J.F.; Heneghan, A.F.; Nadimpalli, A.; Hubert, N.; et al. Effects of Diurnal Variation of Gut Microbes and High-Fat Feeding on Host Circadian Clock Function and Metabolism. Cell Host Microbe 2015, 17, 681-689, doi:10.1016/j.chom.2015.03.006.

55. Liang, X.; Bushman, F.D.; FitzGerald, G.A. Rhythmicity of the Intestinal Microbiota Is Regulated by Gender and the Host Circadian Clock. Proc. Natl. Acad. Sci. 2015, 112, 10479-10484, doi:10.1073/pnas.1501305112.

56. Takada, M.; Nishida, K.; Gondo, Y.; Kikuchi-Hayakawa, H.; Ishikawa, H.; Suda, K.; Kawai, M.; Hoshi, R.; Kuwano, Y.; Miyazaki, K.; et al. Beneficial Effects of Lactobacillus Casei Strain Shirota on Academic Stress-Induced Sleep Disturbance in Healthy Adults: A Double-Blind, Randomised, Placebo-Controlled Trial. Benef. Microbes 2017, 8, 153162, doi:10.3920/BM2016.0150.

57. Miyazaki, K.; Itoh, N.; Yamamoto, S.; Higo-Yamamoto, S.; Nakakita, Y.; Kaneda, H.; Shigyo, T.; Oishi, K. Dietary Heat-Killed Lactobacillus Brevis SBC8803 Promotes Voluntary Wheel-Running and Affects Sleep Rhythms in Mice. Life Sci. 2014, 111, 4752, doi:10.1016/j.Ifs.2014.07.009.

58. Yu, L.; Han, X.; Cen, S.; Duan, H.; Feng, S.; Xue, Y.; Tian, F.; Zhao, J.; Zhang, H.; Zhai, Q.; et al. Beneficial Effect of GABA-Rich Fermented Milk on Insomnia Involving Regulation of Gut Microbiota. Microbiol. Res. 2020, 233, 126409, doi:10.1016/j.micres.2020.126409.

59. Thompson, R.S.; Vargas, F.; Dorrestein, P.C.; Chichlowski, M.; Berg, B.M.; Fleshner, M. Dietary Prebiotics Alter Novel Microbial Dependent Fecal Metabolites That Improve Sleep. Sci. Rep. 2020, 10, 3848, doi:10.1038/s41598-020-60679-y.

60. Staples, A.D.; Bates, J.E.; Petersen, I.T.; McQuillan, M.E.; Hoyniak, C. Measuring Sleep in Young Children and Their Mothers: Identifying Actigraphic Sleep Composites. Int. J. Behav. Dev. 2019, 43, 278-285, doi:10.1177/0165025419830236.

61. Schoch, S.F.; Jenni, O.G.; Kohler, M.; Kurth, S. Actimetry in Infant Sleep Research: An Approach to Facilitate Comparability. Sleep 2019, 42, doi:10.1093/sleep/zsz083.

62. Werner, H.; Molinari, L.; Guyer, C.; Jenni, O.G. Agreement Rates Between Actigraphy, Diary, and Questionnaire for Children's Sleep Patterns. Arch. Pediatr. Adolesc. Med. 2008, 162, 350-358, doi:10.1001/archpedi.162.4.350.

63. Squires, J.; Potter, L.; Bricker, D. The ASQ User's Guide for the Ages \& Stages Questionnaires: A Parent-Completed, Child-Monitoring System; The ASQ user's guide for the Ages \& Stages Questionnaires: A parent-completed, child-monitoring system; Paul H Brookes Publishing: Baltimore, MD, US, 1995; ISBN 978-1-55766-179-1.

64. Sadeh, A.; Acebo, C.; Seifer, R.; Aytur, S.; Carskadon, M.A. Activity-Based Assessment of Sleep-Wake Patterns during the 1st Year of Life. Infant Behav. Dev. 1995, 18, 329337, doi:10.1016/0163-6383(95)90021-7.

65. Meltzer, L.J.; Montgomery-Downs, H.E.; Insana, S.P.; Walsh, C.M. Use of Actigraphy for Assessment in Pediatric Sleep Research. Sleep Med. Rev. 2012, 16, 463-475, doi:10.1016/j.smrv.2011.10.002.

66. Phillips, A.J.; Clerx, W.M.; O’Brien, C.S.; Sano, A.; Barger, L.K.; Picard, R.W.; Lockley, S.W.; Klerman, E.B.; Czeisler, C.A. Irregular Sleep/Wake Patterns Are Associated with 
Poorer Academic Performance and Delayed Circadian and Sleep/Wake Timing. Sci. Rep. 2017, 7, 1-13.

67. Ovreås, L.; Forney, L.; Daae, F.L.; Torsvik, V. Distribution of Bacterioplankton in Meromictic Lake Saelenvannet, as Determined by Denaturing Gradient Gel Electrophoresis of PCR-Amplified Gene Fragments Coding for 16S RRNA. Appl. Environ. Microbiol. 1997, 63, 3367-3373.

68. Krych, t.; Kot, W.; Bendtsen, K.M.B.; Hansen, A.K.; Vogensen, F.K.; Nielsen, D.S. Have You Tried Spermine? A Rapid and Cost-Effective Method to Eliminate Dextran Sodium Sulfate Inhibition of PCR and RT-PCR. J. Microbiol. Methods 2018, 144, 1-7, doi:10.1016/j.mimet.2017.10.015.

69. Andrews, S. FastQC: A Quality Control Tool for High Throughput Sequence Data; Babraham Bioinformatics, Babraham Institute, Cambridge, United Kingdom, 2010;

70. Shen, W.; Le, S.; Li, Y.; Hu, F. SeqKit: A Cross-Platform and Ultrafast Toolkit for FASTA/Q File Manipulation. PloS One 2016, 11, e0163962.

71. Magoč, T.; Salzberg, S.L. FLASH: Fast Length Adjustment of Short Reads to Improve Genome Assemblies. Bioinformatics 2011, 27, 2957-2963.

72. Martin, M. Cutadapt Removes Adapter Sequences from High-Throughput Sequencing Reads. EMBnet J. 2011, 17, 10-12.

73. Schmieder, R.; Edwards, R. Quality Control and Preprocessing of Metagenomic Datasets. Bioinformatics 2011, 27, 863-864.

74. Edgar, R.C.; Flyvbjerg, H. Error Filtering, Pair Assembly and Error Correction for nextGeneration Sequencing Reads. Bioinformatics 2015, 31, 3476-3482, doi:10.1093/bioinformatics/btv401.

75. Edgar, R.C. SINTAX: A Simple Non-Bayesian Taxonomy Classifier for $16 \mathrm{~S}$ and ITS Sequences. BioRxiv 2016, 074161.

76. DeSantis, T.Z.; Hugenholtz, P.; Larsen, N.; Rojas, M.; Brodie, E.L.; Keller, K.; Huber, T.; Dalevi, D.; Hu, P.; Andersen, G.L. Greengenes, a Chimera-Checked 16S RRNA Gene Database and Workbench Compatible with ARB. Appl. Environ. Microbiol. 2006, 72, 5069-5072.

77. Hagerty, S.L.; Hutchison, K.E.; Lowry, C.A.; Bryan, A.D. An Empirically Derived Method for Measuring Human Gut Microbiome Alpha Diversity: Demonstrated Utility in Predicting Health-Related Outcomes among a Human Clinical Sample. PLOS ONE 2020, 15, e0229204, doi:10.1371/journal.pone.0229204.

78. Kong, F.; Hua, Y.; Zeng, B.; Ning, R.; Li, Y.; Zhao, J. Gut Microbiota Signatures of Longevity. Curr. Biol. 2016, 26, R832-R833, doi:10.1016/j.cub.2016.08.015.

79. Arumugam, M.; Raes, J.; Pelletier, E.; Le Paslier, D.; Yamada, T.; Mende, D.R.; Fernandes, G.R.; Tap, J.; Bruls, T.; Batto, J.-M.; et al. Enterotypes of the Human Gut Microbiome. Nature 2011, 473, 174-180, doi:10.1038/nature09944.

80. Gollenberg, A.L.; Lynch, C.D.; Jackson, L.W.; McGuinness, B.M.; Msall, M.E. Concurrent Validity of the Parent-Completed Ages and Stages Questionnaires, 2nd Ed. with the Bayley Scales of Infant Development II in a Low-Risk Sample. Child Care Health Dev. 2010, 36, 485-490, doi:10.1111/j.1365-2214.2009.01041.x.

81. Valla, L.; Wentzel-Larsen, T.; Hofoss, D.; Slinning, K. Prevalence of Suspected Developmental Delays in Early Infancy: Results from a Regional Population-Based Longitudinal Study. BMC Pediatr. 2015, 15, 215, doi:10.1186/s12887-015-0528-z.

82. Bache, S.M.; Wickham, H. Magrittr: A Forward-Pipe Operator for R; 2014;

83. Bates, D.; Sarkar, D.; Bates, M.D.; Matrix, L. The Lme4 Package; 2007; 
84. Becker, J.P.; Knowles, J.E.; Knowles, M.J.E. Package 'Eeptools'; 2020;

85. Bliese, P.; Bliese, M.P. Package 'Multilevel'; 2016;

86. Buuren, S. van; Groothuis-Oudshoorn, K. Mice: Multivariate Imputation by Chained Equations in R. J. Stat. Softw. 2011, 45, 1-67, doi:10.18637/jss.v045.i03.

87. Dixon, P. VEGAN, a Package of R Functions for Community Ecology. J. Veg. Sci. 2003, 14, 927-930.

88. Dowle, M.; Srinivasan, A. Data. Table: Extension Ofdata. Frame. R Package Version 1.12. 2; ed, 2019;

89. Grolemund, G.; Wickham, H. Dates and Times Made Easy with Lubridate. J. Stat. Softw. 2011, 40, 1-25.

90. Hennig, C. Fpc: Flexible Procedures for Clustering. R Package Version 2.1-5; 2013;

91. James, D.; Hornik, K. Chron: Chronological Objects Which Can Handle Dates and Times, 2010;

92. Kassambara, A.; Mundt, F. Factoextra: Extract and Visualize the Results of Multivariate Data Analyses R Package Version 1.0. 5; 2017; 2019;

93. Kassambara, A. Ggpubr:"Ggplot2" Based Publication Ready Plots. R Package Version $012018,7$.

94. Kowarik, A.; Templ, M. Imputation with the R Package VIM. J. Stat. Softw. 2016, 74, 116.

95. Lahti, L.; Shetty, S. Introduction to the Microbiome R Package; 2018;

96. Liaw, A.; Wiener, M. Classification and Regression by RandomForest. $R$ News 2002, 2, 18-22.

97. Lüdecke, D. SjPlot: Data Visualization for Statistics in Social Science; 2018;

98. Maechler, M.; Rousseeuw, P.; Struyf, A.; Hubert, M.; Hornik, K. Cluster: Cluster Analysis Basics and Extensions. R Package Version 2.0. 9. 2019.

99. McMurdie, P.J.; Holmes, S. Phyloseq: An R Package for Reproducible Interactive Analysis and Graphics of Microbiome Census Data. PloS One 2013, 8, e61217.

100. Murrell, P.; Wen, Z. GridGraphics: Redraw Base Graphics Using "grid" Graphics; 2020;

101. Navarro, D. Learning Statistics with $r$ : A Tutorial for Psychology Students and Other Beginners: Version 0.5; University of Adelaide Adelaide, Australia, 2013;

102. Neuwirth, E. RColorBrewer: ColorBrewer Palettes. R Package Version 1.1-2; 2014;

103. Pinheiro, J.; Bates, D.; DebRoy, S.; Sarkar, D.; Heisterkamp, S.; Van Willigen, B.; Maintainer, R. Package 'Nlme'; 2017;

104. Robitzsch, A.; Grund, S.; Henke, T.; Robitzsch, M.A. Package 'Miceadds'; 2017;

105. Sarkar, D. Lattice: Multivariate Data Visualization with R; Springer Science \& Business Media, 2008;

106. Sievert, C. Plotly for R; 2018;

107. Tang, Y.; Horikoshi, M.; Li, W. Ggfortify: Unified Interface to Visualize Statistical Results of Popular R Packages. R J 2016, 8, 474.

108. Wei, T.; Simko, V. R Package "Corrplot": Visualization of a Correlation Matrix (Version 0.84). Retrived Httpsgithub Comtaiyuncorrplot 2017.

109. Wickham, H.; Henry, L. Tidyr: Tidy Messy Data. R Package Version 1.0. 2; 2019;

110. Wickham, H. Reshaping Data with the Reshape Package. J. Stat. Softw. 2007, 21, 1-20.

111. Wickham, H. Stringr: Modern, Consistent String Processing. $R$ J. 2010, 2, 38-40.

112. Wickham, H. Ggplot2: Elegant Graphics for Data Analysis; springer, 2016;

113. Wickham, H.; Francois, R.; Henry, L.; Müller, K. Dplyr: A Grammar of Data Manipulation; 2015; 
114. Wilke, C.O. Cowplot: Streamlined Plot Theme and Plot Annotations for "Ggplot2". $R$ Package Version 0.9. 4; 2019;

115. Xie, Y. Knitr: A General-Purpose Tool for Dynamic Report Generation in R; 2013;

116. Zhu, H. KableExtra: Construct Complex Table with'kable'and Pipe Syntax. URL HttpsCRAN R-Proj. Orgpackage KableExtra R Package Version 0902018.

117. Hamaker, E.L.; Kuiper, R.M.; Grasman, R.P.P.P. A Critique of the Cross-Lagged Panel Model. Psychol. Methods 2015, 20, 102-116, doi:10.1037/a0038889.

118. Bäckhed, F.; Ley, R.E.; Sonnenburg, J.L.; Peterson, D.A.; Gordon, J.I. Host-Bacterial Mutualism in the Human Intestine. Science 2005, 307, 1915-1920, doi:10.1126/science.1104816.

119. Odamaki, T.; Kato, K.; Sugahara, H.; Hashikura, N.; Takahashi, S.; Xiao, J.; Abe, F.; Osawa, R. Age-Related Changes in Gut Microbiota Composition from Newborn to Centenarian: A Cross-Sectional Study. BMC Microbiol. 2016, 16, 90, doi:10.1186/s12866-016-0708-5.

120. Bäckhed, F.; Roswall, J.; Peng, Y.; Feng, Q.; Jia, H.; Kovatcheva-Datchary, P.; Li, Y.; Xia, Y.; Xie, H.; Zhong, H.; et al. Dynamics and Stabilization of the Human Gut Microbiome during the First Year of Life. Cell Host Microbe 2015, 17, 690-703, doi:10.1016/j.chom.2015.04.004.

121. Nagpal, R.; Yamashiro, Y. Gut Microbiota Composition in Healthy Japanese Infants and Young Adults Born by C-Section. Ann. Nutr. Metab. 2018, 73, 4-11, doi:10.1159/000490841.

122. Kuang, Y.-S.; Li, S.-H.; Guo, Y.; Lu, J.-H.; He, J.-R.; Luo, B.-J.; Jiang, F.-J.; Shen, H.; Papasian, C.J.; Pang, H.; et al. Composition of Gut Microbiota in Infants in China and Global Comparison. Sci. Rep. 2016, 6, 36666, doi:10.1038/srep36666.

123. Kostic, A.D.; Gevers, D.; Siljander, H.; Vatanen, T.; Hyötyläinen, T.; Hämäläinen, A.-M.; Peet, A.; Tillmann, V.; Pöhö, P.; Mattila, I.; et al. The Dynamics of the Human Infant Gut Microbiome in Development and in Progression toward Type 1 Diabetes. Cell Host Microbe 2015, 17, 260-273, doi:10.1016/j.chom.2015.01.001.

124. Riggins, T.; Spencer, R.M.C. Habitual Sleep Is Associated with Both Source Memory and Hippocampal Subfield Volume during Early Childhood. Sci. Rep. 2020, 10, 15304, doi:10.1038/s41598-020-72231-z.

125. Kurth, S.; Lassonde, J.M.; Pierpoint, L.A.; Rusterholz, T.; Jenni, O.G.; McClain, I.J.; Achermann, P.; LeBourgeois, M.K. Development of Nap Neurophysiology: Preliminary Insights into Sleep Regulation in Early Childhood. J. Sleep Res. 2016, 25, 646-654, doi:10.1111/jsr.12427.

126. Anders, T.F.; Keener, M. Developmental Course of Nighttime Sleep-Wake Patterns in Full-Term and Premature Infants During the First Year of Life. I. Sleep 1985, 8, 173192, doi:10.1093/sleep/8.3.173.

127. Pennestri, M.-H.; Burdayron, R.; Kenny, S.; Béliveau, M.-J.; Dubois-Comtois, K. Sleeping through the Night or through the Nights? Sleep Med. 2020, doi:10.1016/j.sleep.2020.10.005.

128. Wanqi, S.; Xin, L.S.; Yanrui, J.; Xiaojuan, X.; Karen, S.; Qi, Z.; Chia-huei, T.; Fan, J. A Community-Based Study of Sleep and Cognitive Development in Infants and Toddlers. J. Clin. Sleep Med. 14, 977-984, doi:10.5664/jcsm.7164.

129. Tonetti, L.; Scher, A.; Atun-Einy, O.; Samuel, M.; Boreggiani, M.; Natale, V. Actigraphic Motor Activity during Sleep from Infancy to Adulthood. Chronobiol. Int. 2017, 34, 246-253, doi:10.1080/07420528.2016.1219362. 
130. Tiriac, A.; Sokoloff, G.; Blumberg, M.S. Myoclonic Twitching and Sleep-Dependent Plasticity in the Developing Sensorimotor System. Curr. Sleep Med. Rep. 2015, 1, 7479, doi:10.1007/s40675-015-0009-9.

131. Blumberg, M.S.; Marques, H.G.; lida, F. Twitching in Sensorimotor Development from Sleeping Rats to Robots. Curr. Biol. 2013, 23, R532-R537, doi:10.1016/j.cub.2013.04.075.

132. Rio-Bermudez, C.D.; Blumberg, M.S. Active Sleep Promotes Functional Connectivity in Developing Sensorimotor Networks. BioEssays 2018, 40, 1700234, doi:10.1002/bies.201700234.

133. Seehagen, S.; Konrad, C.; Herbert, J.S.; Schneider, S. Timely Sleep Facilitates Declarative Memory Consolidation in Infants. Proc. Natl. Acad. Sci. 2015, 112, 16251629, doi:10.1073/pnas.1414000112.

134. Touchette, É.; Petit, D.; Séguin, J.R.; Boivin, M.; Tremblay, R.E.; Montplaisir, J.Y. Associations Between Sleep Duration Patterns and Behavioral/Cognitive Functioning at School Entry. Sleep 2007, 30, 1213-1219, doi:10.1093/sleep/30.9.1213.

135. Mullen, E.M. Mullen Scales of Early Learning; AGS Circle Pines, MN, 1995;

136. Ball, H.L.; Douglas, P.S.; Kulasinghe, K.; Whittingham, K.; Hill, P. The Possums Infant Sleep Program: Parents' Perspectives on a Novel Parent-Infant Sleep Intervention in Australia. Sleep Health 2018, 4, 519-526, doi:10.1016/j.sleh.2018.08.007.

137. Radke, M.; Picaud, J.-C.; Loui, A.; Cambonie, G.; Faas, D.; Lafeber, H.N.; de Groot, N.; Pecquet, S.S.; Steenhout, P.G.; Hascoet, J.-M. Starter Formula Enriched in Prebiotics and Probiotics Ensures Normal Growth of Infants and Promotes Gut Health: A Randomized Clinical Trial. Pediatr. Res. 2017, 81, 622-631, doi:10.1038/pr.2016.270. 
Table 1. Associations between alpha diversity and sleep behavior in the first year of life.

\begin{tabular}{|c|c|c|c|c|c|c|c|c|}
\hline \multirow[b]{2}{*}{ Shannon } & \multicolumn{2}{|l|}{ Overall } & \multicolumn{2}{|l|}{3 Months } & \multicolumn{2}{|l|}{6 Months } & \multicolumn{2}{|l|}{12 Months } \\
\hline & $\begin{array}{l}\text { Estimate } \pm \\
\text { SEM }\end{array}$ & $P$ value & Estimate \pm SEM & $P$ value & Estimate \pm SEM & $P$ value & Estimate \pm SEM & $P$ value \\
\hline (Intercept) & $1.55 \pm 0.15$ & $<0.001$ & $2.51 \pm 0.76$ & $<0.001$ & $-0.08 \pm 0.98$ & 0.94 & $7.22 \pm 2.57$ & 0.01 \\
\hline Exact_Age & $0.1 \pm 0.02$ & $<0.001$ & $-0.25 \pm 0.26$ & 0.33 & $0.38 \pm 0.17$ & 0.03 & $-0.36 \pm 0.22$ & 0.10 \\
\hline RunSecond & $-0.09 \pm 0.09$ & 0.31 & $-0.15 \pm 0.14$ & 0.26 & $-0.06 \pm 0.14$ & 0.66 & $-0.19 \pm 0.21$ & 0.37 \\
\hline RunThird & $-0.11 \pm 0.09$ & 0.22 & $-0.06 \pm 0.13$ & 0.65 & $-0.11 \pm 0.15$ & 0.47 & $-0.27 \pm 0.22$ & 0.22 \\
\hline RunFourth & $-0.29 \pm 0.08$ & $<0.001$ & $-0.31 \pm 0.14$ & 0.02 & $-0.26 \pm 0.12$ & 0.03 & $-0.47 \pm 0.2$ & 0.02 \\
\hline RunFifth & $-0.05 \pm 0.11$ & 0.66 & $0.49 \pm 0.41$ & 0.23 & $-0.27 \pm 0.23$ & 0.24 & $-0.12 \pm 0.2$ & 0.54 \\
\hline sexfemale & $-0.05 \pm 0.06$ & 0.38 & $-0.11 \pm 0.1$ & 0.27 & $-0.09 \pm 0.1$ & 0.34 & $0.05 \pm 0.1$ & 0.63 \\
\hline BreastFeeding_Yes & $-0.13 \pm 0.09$ & 0.12 & - & - & $-0.12 \pm 0.18$ & 0.53 & $-0.2 \pm 0.11$ & 0.07 \\
\hline Sleep_Activity & $0.02 \pm 0.03$ & 0.66 & $0.06 \pm 0.06$ & 0.28 & $-0.01 \pm 0.06$ & 0.92 & $0.01 \pm 0.07$ & 0.86 \\
\hline Sleep_Day & $-0.09 \pm 0.05$ & 0.08 & $-0.2 \pm 0.09$ & 0.04 & $-0.15 \pm 0.1$ & 0.12 & $0.04 \pm 0.09$ & 0.64 \\
\hline Sleep_Night & $0 \pm 0.03$ & 0.98 & $-0.02 \pm 0.05$ & 0.73 & $-0.04 \pm 0.05$ & 0.40 & $0.05 \pm 0.06$ & 0.46 \\
\hline Sleep_Timing & $0.01 \pm 0.03$ & 0.86 & $0.03 \pm 0.05$ & 0.58 & $-0.02 \pm 0.06$ & 0.78 & $0.04 \pm 0.07$ & 0.56 \\
\hline Sleep_Variability & $\begin{array}{r}-0.02 \pm 0.03 \\
\text { Estimate } \pm\end{array}$ & 0.62 & $0.01 \pm 0.05$ & 0.83 & $-0.1 \pm 0.06$ & 0.14 & $-0.03 \pm 0.05$ & 0.64 \\
\hline Observed overall & SEM & $P$ value & Estimate \pm SEM & $P$ value & Estimate \pm SEM & $P$ value & Estimate \pm SEM & $P$ value \\
\hline (Intercept) & $76.6 \pm 12.16$ & $<0.001$ & $112.18 \pm 51.91$ & 0.03 & $-27.47 \pm 73.19$ & 0.71 & $628.97 \pm 228.06$ & 0.01 \\
\hline Exact_Age & $10.94 \pm 1.3$ & $<0.001$ & $-0.49 \pm 17.65$ & 0.98 & $28.16 \pm 12.29$ & 0.02 & $-34.77 \pm 19.58$ & 0.08 \\
\hline RunSecond & $-7.7 \pm 6.65$ & 0.25 & $-12.4 \pm 9.81$ & 0.21 & $2.06 \pm 10.49$ & 0.84 & $-17.78 \pm 18.6$ & 0.34 \\
\hline RunThird & $-2.35 \pm 6.7$ & 0.73 & $3.95 \pm 8.46$ & 0.64 & $-6.89 \pm 11.19$ & 0.54 & $-12.27 \pm 19.38$ & 0.53 \\
\hline
\end{tabular}




\begin{tabular}{|c|c|c|c|c|c|c|c|c|}
\hline RunFourth & $-16.48 \pm 6.35$ & 0.01 & $-14.47 \pm 9.25$ & 0.12 & $-10.77 \pm 8.73$ & 0.22 & $-30.01 \pm 17.91$ & 0.10 \\
\hline RunFifth & $-2.91 \pm 8.45$ & 0.73 & $10.17 \pm 27.08$ & 0.71 & $-7.53 \pm 17.01$ & 0.66 & $-9.04 \pm 17.66$ & 0.61 \\
\hline sexfemale & $-1.04 \pm 4.83$ & 0.83 & $1.08 \pm 6.59$ & 0.87 & $-0.64 \pm 7.08$ & 0.93 & $-0.32 \pm 8.57$ & 0.97 \\
\hline Sleep_Activity & $1.4 \pm 2.73$ & 0.61 & $0.87 \pm 3.85$ & 0.82 & $4.75 \pm 4.59$ & 0.30 & $-4.13 \pm 6.77$ & 0.54 \\
\hline Sleep_Day & $-8.57 \pm 4$ & 0.03 & $-20.36 \pm 6.9$ & 0.004 & $-4.04 \pm 7.02$ & 0.57 & $0.32 \pm 7.81$ & 0.97 \\
\hline $\begin{array}{l}\text { Sleep_Variability } \\
\text { Chao1 Overall }\end{array}$ & $\begin{array}{c}-0.7 \pm 2.51 \\
\text { Estimate } \pm \\
\text { SEM }\end{array}$ & $P$ value & $\begin{array}{l}6.34 \pm 3.71 \\
\text { Estimate } \pm \text { SEM }\end{array}$ & $P$ value & $\begin{array}{l}-1.66 \pm 4.65 \\
\text { Estimate } \pm \text { SEM }\end{array}$ & $P$ value & $\begin{array}{l}-7.85 \pm 4.92 \\
\text { Estimate } \pm \text { SEM }\end{array}$ & $P$ value \\
\hline (Intercept) & $\begin{array}{l}96.47 \pm \\
13.42\end{array}$ & $<0.001$ & $124.28 \pm 59.19$ & 0.04 & $-39.76 \pm 86.41$ & 0.65 & $808.4 \pm 251.59$ & $<0.001$ \\
\hline RunFourth & $-19.27 \pm 7.24$ & 0.01 & $-19.76 \pm 10.83$ & 0.07 & $-9.93 \pm 10.58$ & 0.35 & $-37.07 \pm 19.63$ & 0.06 \\
\hline RunFifth & $-10.56 \pm 9.85$ & 0.28 & $0.55 \pm 31.37$ & 0.99 & $-10.4 \pm 19.72$ & 0.60 & $-18.73 \pm 19.38$ & 0.34 \\
\hline sexfemale & $0.42 \pm 5.45$ & 0.94 & $6.5 \pm 7.59$ & 0.39 & $-2.18 \pm 8.7$ & 0.80 & $1.06 \pm 9.37$ & 0.91 \\
\hline BreastFeeding_Yes & $-5.82 \pm 8.13$ & 0.47 & - & & $-2.49 \pm 16.4$ & 0.88 & $-10.56 \pm 10.68$ & 0.32 \\
\hline Sleep_Activity & $1.58 \pm 3.12$ & 0.61 & $-0.25 \pm 4.43$ & 0.96 & $6.64 \pm 5.54$ & 0.23 & $-4.59 \pm 7.22$ & 0.53 \\
\hline Sleep_Day & $-9.73 \pm 4.25$ & 0.02 & $-20.55 \pm 7.53$ & 0.01 & $-5.31 \pm 8.54$ & 0.54 & $0.29 \pm 8.26$ & 0.97 \\
\hline Sleep_Night & $-0.62 \pm 2.65$ & 0.82 & $-5.75 \pm 3.71$ & 0.12 & $0.09 \pm 4.53$ & 0.98 & $0.18 \pm 6.2$ & 0.98 \\
\hline Sleep_Timing & $-1.58 \pm 3$ & 0.60 & $-3.98 \pm 4.14$ & 0.34 & $-2.22 \pm 4.89$ & 0.65 & $6.23 \pm 6.45$ & 0.34 \\
\hline
\end{tabular}


Note. Standard errors of the mean are reported. Breastfeeding was not included in the model at 3 months because all infants were (over $50 \%$ ) breastfed at that age. Bold shows significant associations $(P<0.05)$. 
Table 2. Associations between the Bacterial Maturation Index and sleep composites across the first year of life.

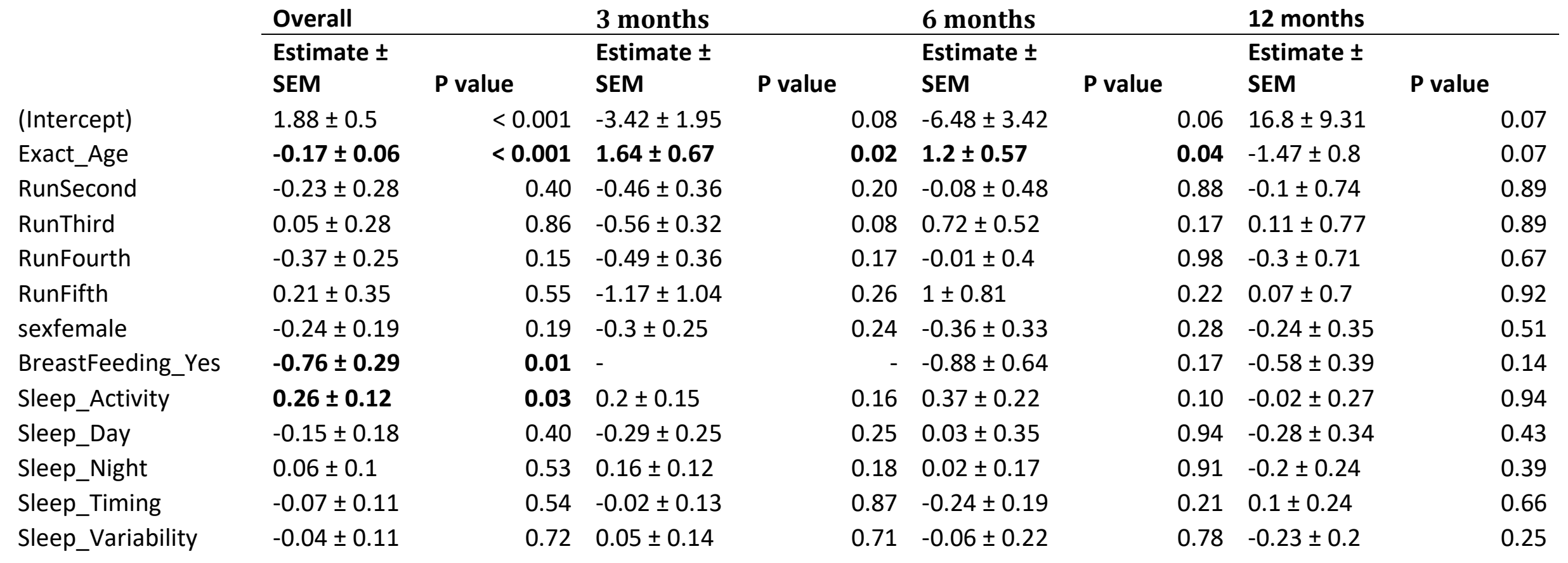

Note. Standard errors of the mean are reported. Breastfeeding was not included in the model at 3 months because all infants were (over $50 \%$ ) breastfed at that age. Bold marks significant associations $(P<0.05)$. 
Table 3. Associations between infant enterotype (A or B) with sleep variables in the first year of life.

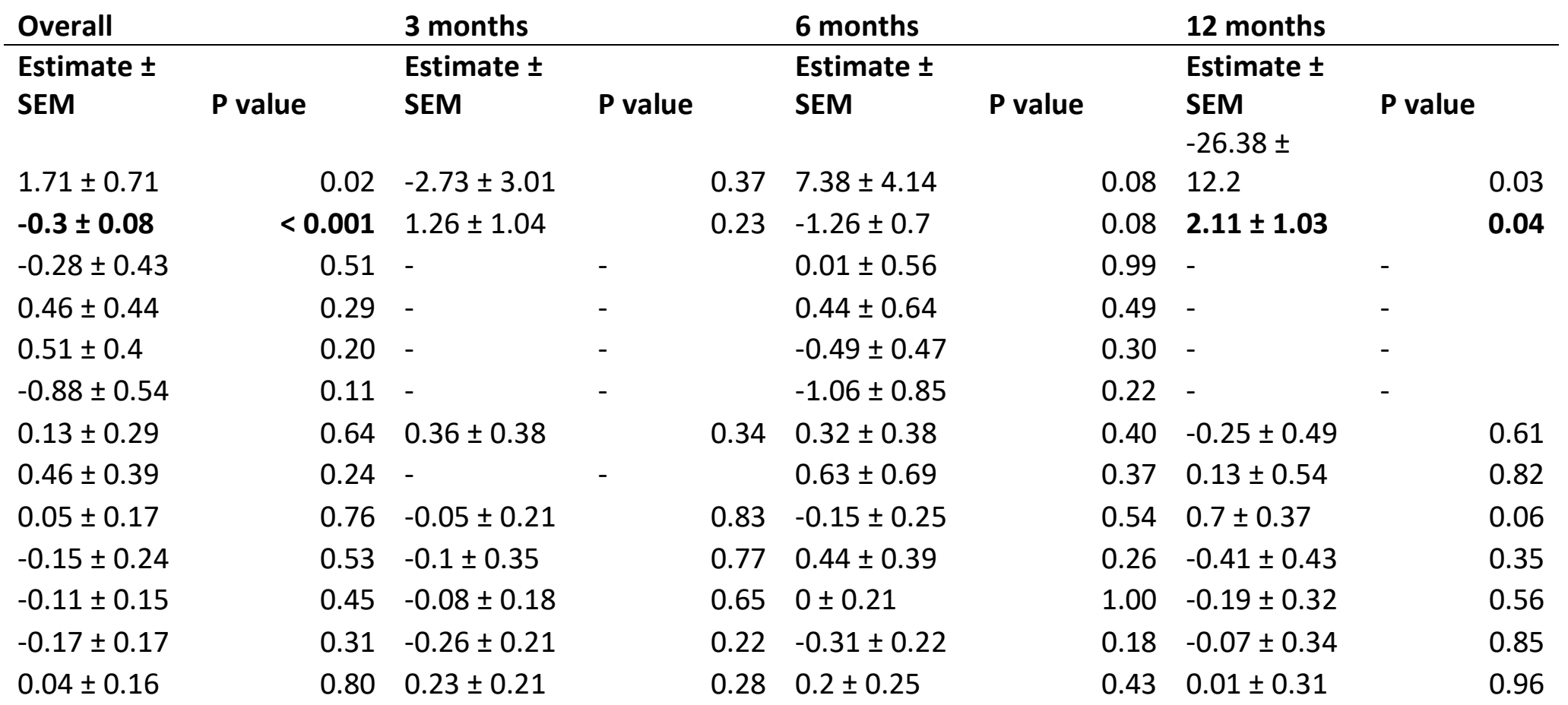

Note. Standard errors of the mean are reported. Breastfeeding was not included in the model at 3 months because all infants were (over $50 \%$ ) breastfed at that age. Run could not be included for models at 3 and 12 months because not all combinations of Run/Enterotype existed at these timepoints. Positive estimates signify associations with enterotype A. Enterotype A is linked with higher abundance of Bifidobacterium while enterotype $B$ is linked with higher abundance of Bacteroides. Bold marks significant associations $(P<0.05)$. 